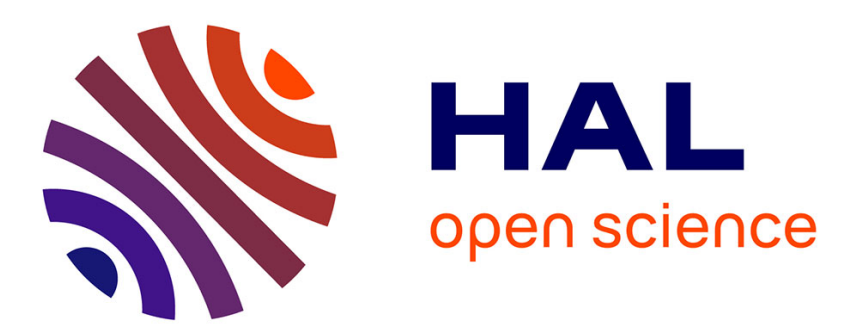

\title{
Solutal buoyancy and electrovortex flow in liquid metal batteries
}

\author{
W. Herreman, S. Bénard, Caroline Nore, P. Personnettaz, L. Cappanera, J.-L. \\ Guermond
}

\section{- To cite this version:}

W. Herreman, S. Bénard, Caroline Nore, P. Personnettaz, L. Cappanera, et al.. Solutal buoyancy and electrovortex flow in liquid metal batteries. Physical Review Fluids, 2020, 5 (7), pp.074501. 10.1103/PhysRevFluids.5.074501 . hal-03335872

\section{HAL Id: hal-03335872 \\ https://hal.science/hal-03335872}

Submitted on 6 Sep 2021

HAL is a multi-disciplinary open access archive for the deposit and dissemination of scientific research documents, whether they are published or not. The documents may come from teaching and research institutions in France or abroad, or from public or private research centers.
L'archive ouverte pluridisciplinaire HAL, est destinée au dépôt et à la diffusion de documents scientifiques de niveau recherche, publiés ou non, émanant des établissements d'enseignement et de recherche français ou étrangers, des laboratoires publics ou privés. 


\title{
Solutal buoyancy and electrovortex flow in liquid metal batteries
}

\author{
W. Herreman $\odot^{, *}$ S. Bénard, and C. Nore $\odot$ \\ Laboratoire d'Informatique pour la Mécanique et les Sciences de l'Ingénieur, LIMSI, CNRS, Université \\ Paris-Saclay, Bât 507, Campus Universitaire F-91405 Orsay, France \\ P. Personnettaz $(0)$ \\ Helmholtz-Zentrum Dresden-Rossendorf, 01328 Dresden, Germany \\ L. Cappanera $\odot$ \\ Department of Mathematics, University of Houston, Houston, Texas 77204-3008, USA \\ J.-L. Guermond $\odot$ \\ Department of Mathematics, Texas A\&M University 3368 TAMU, College Station, Texas 77843-3368, USA
}

(Received 21 November 2019; accepted 24 June 2020; published 20 July 2020)

\begin{abstract}
Solutal buoyancy has a large impact on the flow of the alloy phase composing the positive electrode in liquid metal batteries. During discharge solutal buoyancy creates a stabilizing stratification, during charge it creates a vigorous solutal convection. In this article we provide new physical understandings of the role of solutal buoyancy during both charge and discharge. In particular we find that during discharge the electrovortex mechanism is in general not strong enough to counter the stabilizing effect of solutal buoyancy, and therefore this mechanism cannot be used to mix the alloy as is sometimes suggested in the literature. We show that the mixing capability of a generic flow in the alloy phase can be estimated by comparing the typical flow magnitude $U$ to two velocity scales: $U_{p}$ and $U_{m}$. Below $U_{p}$ the flow cannot mix the alloy, and above $U_{m}$ the flow significantly opposes solutal buoyancy. Although we focus on $\mathrm{Li}|| \mathrm{Pb}$-based batteries, these simple mixing criteria can be used during the discharging phase in other types of liquid batteries. We also present new, fully three-dimensional simulations of solutal convection during the charging cycle. These simulations suggest scaling laws for the magnitude of the convective flow, the time for the onset of solutal convection, and the typical inhomogeneity level in the alloy during charge. We propose physical arguments to explain these scaling laws.
\end{abstract}

DOI: 10.1103/PhysRevFluids.5.074501

\section{INTRODUCTION}

Liquid metal batteries (LMBs) are a particular type of high-temperature electrochemical cells composed of three layers of superposed liquids. As shown in Fig. 1(a), there is a light liquid metal (e.g., Li) sitting on top of a heavier molten salt, which itself sits on top of a heavier alloy (e.g., Li-Pb). We refer to the top layer as the negative electrode, the middle layer as the electrolyte, and the bottom layer as the positive electrode. The technology is not new [1,2] but the possibility of producing very large cells that can be made of Earth-abundant materials [3-5] makes LMBs interesting candidates for large-scale stationary storage of electrical energy.

\footnotetext{
*wietze.herreman@universite-paris-saclay.fr
} 


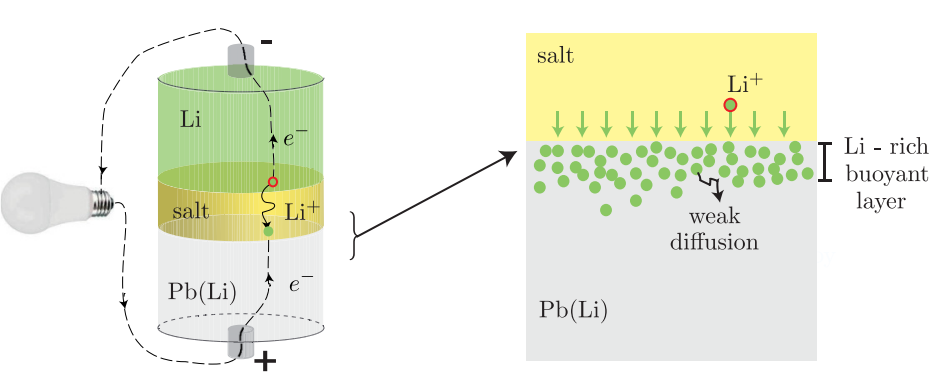

(a)

(b)

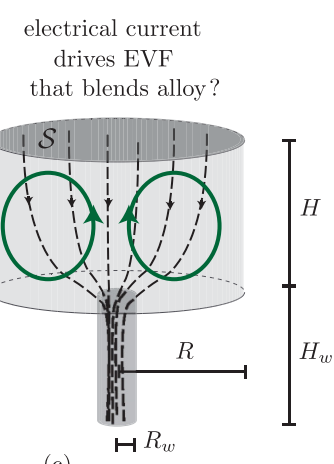

(c)

FIG. 1. Discharge process in a $\mathrm{Li} \| \mathrm{Pb}$ liquid metal battery: (a) $\mathrm{Li}^{+}$ions move across the molten salt electrolyte toward the bottom $\mathrm{Li}-\mathrm{Pb}$ alloy composing the positive electrode. (b) In the positive electrode, $\mathrm{Li}$ diffuses weakly and locally accumulates near the interface with the salt in a stably stratified buoyant layer. This layer lowers the battery performance and may trigger the early formation of solid intermetallics. (c) An inhomogeneous electrical current density in the alloy creates an electrovortex flow. One objective of the paper is to investigate whether this flow can mix the alloy during discharge and therefore prevent the formation of the locally Li-enriched layer at the interface separating the alloy and the salt.

Being all liquid, flows of diverse origin can occur in LMBs (see review of Ref. [6]). Although intense fluid motions are undesirable and should be avoided [7], fluid flows can be beneficial to LMBs since they may help homogenize the bottom layer which composes the positive electrode. In Fig. 1(b) we sketch the problem that arises in LMBs that are too rapidly discharged. Just below the interface, the incoming flux of light material (here Li) becomes so large that diffusion alone cannot homogenize the alloy rapidly enough. This results in local accumulation of Li just below the interface. During charge, local depletion of the alloy can similarly occur. Inhomogeneities in the alloy are undesirable for two reasons. First, they affect the potential of the cell which then gets lower during discharge and higher during charge, making the battery less efficient. Second, they can trigger (during discharge) the formation of solid intermetallic (Li-rich) phases that may be harmful to the battery, although this may not always be the case as documented in Ref. [8]. To avoid local inhomogeneities in the alloy during charge and discharge, the homogenization process has to be to accelerated. This can be done, for example, by producing a sufficiently intense flow in the alloy layer. LMBs with naturally occurring mixing flows in the alloy layer are likely to operate with higher current densities. As mentioned in Ref. [9], this idea is not new, but it reemerged recently in the wake of Ref. [10] followed by Refs. [9,11-14].

Of all the possible ways to mix the bottom layer alloy in LMBs, the electrovortex flow mechanism has been suggested in the literature to be a good candidate, likely because it is relatively simple to control. All that is needed for this phenomenon to occur is to have a thin wire connecting to a wider conducting fluid layer as shown in Fig. 1(c). The Lorentz force associated with the deviated electrical current (dashed lines) causes an effective force that blows the alloy away from the thinner electrical contact (as suggested by the pair of loops in the figure). The experimental study in Ref. [10] followed by the numerical simulations in Ref. [11-13] all suggest that the electrovortex mechanism combined with thermal buoyancy can yield a nonnegligible flow in the alloy, but the mixing of the alloy itself, i.e., its spatially and temporally varying composition was not considered in these references. A first attempt to model mixing in the alloy was done in Ref. [11]: The alloy composition was simulated as a passive scalar using a prescribed flow field similar to that produced by the electrovortex mechanism. Recently, Ref. [14] proposed a more complete model for the $\mathrm{Li}|| \mathrm{Bi}$ cell. No electrovortex flow is present in this study, but the varying composition of the alloy and in particular the solutal buoyancy it causes are taken into account. Although neglected in all the previous works on flows in LMBs, it is shown in Ref. [14] that 
the impact of solutal buoyancy on the flow is immense. During charge, when light Li is being depleted, the alloy locally becomes heavier and quickly destablizes the hydrostatic equilibrium, thereby producing a vigorous solutal convection, even in small cells. Interestingly, this also means that solutal buoyancy naturally prevents the formation of large inhomogeneities in the alloy during charge. Solutal buoyancy seriously complexifies the task of mixing the alloy during discharge, since solutal buoyancy stabilizes the alloy vertically when light Li migrates from the electrolyte into the alloy and the only mechanism for $\mathrm{Li}$ atoms to migrate further down in the cell is diffusion. The Li-enriched layer, sketched in Fig. 1(b), can easily become $1000 \mathrm{~kg} \mathrm{~m}^{-3}$ lighter than the bulk alloy if no sufficiently intense flow prevents its formation and persistence. In the recent study in Ref. [9], the authors propose to combine the electrovortex mechanism with an ambient magnetic field and call this setting swirling electrovortex flow. They show that the swirling electrovortex flow can indeed generate a fluid motion which in some circumstances counters solutal buoyancy, but whether this is always the case is not clear.

Overall, we need a better understanding of the role of solutal buoyancy in LMBs. During charge, we know that solutal convection occurs [14], but can we a priori estimate the typical intensity of the flow, the mixing time and the inhomogeneity level in the alloy? In this article, we provide answers to these questions in the form of scaling laws that only depend on the input parameters. These laws are directly inspired from new, fully three-dimensional (3D) simulations of solutal convection in LMBs. During discharge we know that solutal buoyancy stabilizes the hydrostatic equilibrium. To estimate whether the nonswirling electrovortex mechanism can overcome buoyancy and efficiently mix the alloy, we have performed numerical simulations. These simulations suggest that this is not the case in small cells. In the paper we theoretically estimate the flow intensity that is required to overcome solutal buoyancy. We derive two characterstic velocity scales, $U_{p}$ and $U_{m}$, which only depend on the input parameters. Below $U_{p}$ the flow intensity is not strong enough and above $U_{m}$ robust mixing occurs. These estimates can be used to extrapolate mixing strategies to other types of batteries. They can be also used in other flow contexts.

The article is structured as follows. In Sec. 2 we define the mathematical model and the physical characteristics of the LMB under investigation. In Sec. 3 we study the discharge phase of the LMB. In Sec. 4 we study the charge. In Appendix A, we provide supplementary information on the model. In Appendix B, we compare single phase simulations in the bottom layer alone to multiphase simulations of the full LMB. These simulations demonstrate that, in all the situations considered in the paper, the salt-alloy interface is well approximated as an undeformable interface on which the no-slip boundary condition for the velocity applies.

\section{MODEL}

\section{A. Set-up and evolution laws}

In our simulations, we consider a small liquid metal battery with cylindrical shape. Rather than solving the full multiphase configuration with three layers as in Ref. [15], we focus as in Ref. [14] on the bottom layer which is the positive electrode of the battery. This alloy layer has radius $R=4 \mathrm{~cm}$ and fixed height $H=2 \mathrm{~cm}$. In reality, $H$ changes in time during charge and discharge but only very slowly. We use a Boussinesq model to simulate the fluid flow and solve the induction equation for the magnetic field:

$$
\begin{aligned}
\rho_{*}\left[\partial_{t} \boldsymbol{u}+(\boldsymbol{u} \cdot \nabla) \boldsymbol{u}\right] & =-\nabla p-\left[\rho_{*}-\chi\left(\rho_{\mathrm{Li}}-\rho_{\mathrm{Li}, *}\right)\right] g \boldsymbol{e}_{z}+\nabla \cdot\left[\rho_{*} v\left(\nabla \boldsymbol{u}+(\nabla \boldsymbol{u})^{\mathbf{T}}\right)\right]+\boldsymbol{j} \times \boldsymbol{b}, \\
\partial_{t} \boldsymbol{b} & =\nabla \times(\boldsymbol{u} \times \boldsymbol{b})+\left(\mu_{0} \sigma\right)^{-1} \Delta \boldsymbol{b}, \\
\nabla \cdot \boldsymbol{u} & =0, \quad \nabla \cdot \boldsymbol{b}=0 .
\end{aligned}
$$

To keep track of the composition of the alloy, we solve the advection-diffusion problem:

$$
\partial_{t} \rho_{\mathrm{Li}}+\boldsymbol{u} \cdot \nabla \rho_{\mathrm{Li}}=D \nabla \rho_{\mathrm{Li}}
$$


We denote $\boldsymbol{u}$ the velocity, $p$ the pressure, $\boldsymbol{b}$ the magnetic induction, $\boldsymbol{j}=\mu_{0}^{-1} \nabla \times \boldsymbol{b}$ the current density, and $\rho_{\mathrm{Li}}$ the mass concentration of $\mathrm{Li}$ in the alloy. The reference densities $\rho_{*}, \rho_{\mathrm{Li}, *}$ depend on the configuration (charge or discharge) and are specified below, $g$ is the gravity constant, and $\mu_{0}$ is the magnetic permeability.

In order to be able to study solutal convection in the alloy, it is crucial to know how the density $\rho$ varies with the alloy composition. For Li-Pb alloys, Refs. [16,17] provide experimental data for the density $\rho=\rho\left(x_{\mathrm{Li}}\right)$ of the alloy as a function of the molar fraction $x_{\mathrm{Li}}$ of lithium in the alloy. Denoting $\left(\mathcal{M}_{\mathrm{Li}}, \mathcal{M}_{\mathrm{Pb}}\right)=(6.941,207.2) \times 10^{-3} \mathrm{~kg} \mathrm{~mol}^{-1}$ the molar masses of lithium and lead, and using the fundamental relation

$$
\rho_{\mathrm{Li}}=\rho\left(1+\frac{1-x_{\mathrm{Li}}}{x_{\mathrm{Li}}} \frac{\mathcal{M}_{\mathrm{Pb}}}{\mathcal{M}_{\mathrm{Li}}}\right)^{-1},
$$

which connects the molar fraction $x_{\mathrm{Li}}$ to the mass concentration $\rho_{\mathrm{Li}}$ and the density $\rho$, we reexpress the experimental measurements of Ref. [16] as $\rho=\rho\left(\rho_{\mathrm{Li}}\right)$. This is done graphically in Appendix A, and Fig. 14(a) suggests that the following linear fit for the density of Li-Pb alloys

$$
\rho \approx \rho_{*}-\chi\left(\rho_{\mathrm{Li}}-\rho_{\mathrm{Li}, *}\right) \quad \text { with } \quad \chi=-\frac{d \rho}{d \rho_{\mathrm{Li}}} \approx 15.1
$$

is fairly accurate over the entire range $x_{\mathrm{Li}} \in[0,60] \%$. This expression is used in the Boussinesq approximation of the buoyancy force in (1a). The fit (3) is designed to be exact at the eutectic $x_{\mathrm{Li}}=$ $17 \%$ mixture which we use as reference in the numerical simulations of discharge. In the numerical simulations of charge, we suppose that the initial alloy has the molar fraction $x_{\mathrm{Li}}=50 \%$. Hence the reference densities are different depending on whether we consider a charge or a discharge phase:

$$
\begin{gathered}
(\text { discharge }):\left(\rho_{*}, \rho_{\mathrm{Li}, *}\right)=\left(\rho_{17 \%}, \rho_{\mathrm{Li}, 17 \%}\right)=(9543,65) \mathrm{kg} \mathrm{m}^{-3}, \\
(\text { charge }):\left(\rho_{*}, \rho_{\mathrm{Li}, *}\right)=\left(\rho_{50 \%}, \rho_{\mathrm{Li}, 50 \%}\right)=(7066,229) \mathrm{kg} \mathrm{m}^{-3} .
\end{gathered}
$$

Instead of the nondimensional number $\chi$, one can also use the more common solutal expansion coefficient $\beta=\chi / \rho_{*}$ of the Li-Pb alloy. The coefficient $\chi$ is constant but, during charge and during discharge the solutal expansion coefficient $\beta$ is different because the reference densities change:

$$
\begin{aligned}
(\text { discharge) }: \beta & =\chi / \rho_{17 \%}=1.58 \times 10^{-3} \mathrm{~m}^{3} \mathrm{~kg}^{-1}, \\
(\text { charge }): & =\chi / \rho_{50 \%}=2.14 \times 10^{-3} \mathrm{~m}^{3} \mathrm{~kg}^{-1} .
\end{aligned}
$$

Notice that these values are remarkably close to those used in Ref. [14] for a totally different Li-Bi alloy. Although the numerical simulations are done with $\rho_{\mathrm{Li}}$, we prefer to report all the numerical results in the figures with the molar fraction $x_{\mathrm{Li}}$ of the alloy. Combining (2) and (3a) we obtain the following explicit expression connecting $\rho_{\mathrm{Li}}$ to $x_{\mathrm{Li}}$ :

$$
x_{\mathrm{Li}}=\left[1+\frac{\mathcal{M}_{\mathrm{Li}}}{\mathcal{M}_{\mathrm{Pb}}}\left(\frac{\rho_{0 \%}}{\rho_{\mathrm{Li}}}-\chi-1\right)\right]^{-1} \text {. }
$$

In this formula $\rho_{0 \%}=\rho_{17 \%}+\chi \rho_{\mathrm{Li}, 17 \%}=10525 \mathrm{~kg} \mathrm{~m}^{-3}$ is the extrapolated density of pure lead, which is close to the actual density of pure lead $\left(10433 \mathrm{~kg} \mathrm{~m}^{-3}\right)$. In all the numerical simulations, we suppose

$$
v \approx 1.44 \times 10^{-7} \mathrm{~m}^{2} \mathrm{~s}^{-1}, \quad \sigma \approx 7.39 \times 10^{5} \mathrm{~S} \mathrm{~m}^{-1}, \quad D \approx 8 \times 10^{-9} \mathrm{~m}^{2} \mathrm{~s}^{-1},
$$

for the kinematic viscosity, the electrical conductivity, and the binary diffusion coefficient. These values are realistic in the eutectic $x_{\mathrm{Li}}=17 \%$ mixture but are certainly approximative for the $x_{\mathrm{Li}}=$ $50 \%$ alloy for which we have no detailed information. Appendix A gives further details on the material properties of the alloy and also discusses the validity of the evolution equations considered in this work. 
In the numerical simulations, we electrically connect the bottom of the fluid domain to a thin copper wire of radius $R_{w}=0.8 \mathrm{~cm}$, height $H_{w}=2 \mathrm{~cm}$, and electrical conductivity $\sigma_{w}=$ $5.96 \times 10^{7} \mathrm{~S} \mathrm{~m}^{-1}$. The equations we solve for the magnetic induction $\boldsymbol{b}_{w}$ in the copper wire are

$$
\partial_{t} \boldsymbol{b}_{w}=\left(\sigma_{w} \mu_{0}\right)^{-1} \Delta \boldsymbol{b}_{w}, \quad \nabla \cdot \boldsymbol{b}_{w}=0 .
$$

Similar wires of aspect ratio $R_{w} / R=0.2$ were used in Ref. [15].

The above set of equations comes with boundary conditions. For the flow, we suppose that the no-slip condition holds everywhere, even at the top of the fluid domain $z=H$, which is actually the interface with the electrolyte. As shown in Appendix B, the no-slip condition turns out to be quite realistic when compared to multiphase simulations with free interfaces. It is definitely more realistic than the free-slip boundary condition. All the electrical boundary conditions are enforced through the magnetic field, and we follow precisely the same approach as in Ref. [15]. Using Ampère's law

$$
\left.b_{\theta}\right|_{r=r_{b}}=\frac{\mu_{0}}{r_{b}} \int_{0}^{r_{b}} j_{z} r d r
$$

we can calculate $b_{\theta}$ at any point (at some radius $r_{b}$ ) of the boundary. At $z=H$, which is the top of the simulated fluid domain, we suppose that the current density is homogeneous and vertical, $\left.\boldsymbol{j}\right|_{z=H}=J \boldsymbol{e}_{z}$. We are going to vary the current density $J$ in the numerical simulation. Setting $B_{0}=$ $\mu_{0} J R / 2$, Ampère's law gives

$$
\left.b_{\theta}\right|_{z=H}=B_{0} \frac{r}{R}, \quad \forall r \in[0, R] .
$$

This is an approximation that ignores the local variations of the electric current density due to mass transfer and chemical reactions at the interface (see Ref. [18] for a more advanced model that takes these effects into account). On the insulated parts of the boundary of the alloy layer, we set

$$
\left.b_{\theta}\right|_{r=R}=B_{0}, \quad \forall z \in[0, H] ;\left.\quad b_{\theta}\right|_{z=0}=B_{0} \frac{R}{r}, \quad \forall r \in\left[R_{w}, R\right] .
$$

At the electrical contact between the alloy and the wire, the tangential components of the magnetic field and of the electric field are continuous:

$$
\boldsymbol{e}_{z} \times\left.\left(\boldsymbol{b}-\boldsymbol{b}_{w}\right)\right|_{z=0}=0, \quad \boldsymbol{e}_{z} \times\left.\left[(\boldsymbol{j} / \sigma)-\left(\boldsymbol{j}_{w} / \sigma_{w}\right)\right]\right|_{z=0}=0, \quad \forall r \in\left[0, R_{w}\right] .
$$

The cylindrical surface of the wire is insulated, and we suppose that the current density is uniform at the bottom of the wire, $z=-H_{w}$. By conservation of the total current $I=J \pi R^{2}$, we find that $\left.\boldsymbol{j}_{w}\right|_{z=-H_{w}}=25 \mathrm{~J} \boldsymbol{e}_{z}$ since $R / R_{w}=5$. Then, the boundary conditions on the magnetic field on the boundary of the wire are

$$
\left.b_{w, \theta}\right|_{r=R_{w}}=B_{0} \frac{R}{R_{w}}, \quad \forall z \in\left[-H_{w}, 0\right] ;\left.\quad b_{w, \theta}\right|_{z=-H_{w}}=B_{0} \frac{r R}{R_{w}^{2}}, \quad \forall r \in\left[0, R_{w}\right] .
$$

This is all we need in axisymmetric simulations, since $b_{r}=b_{z}=0$ by symmetry. Three-dimensional simulations require additional boundary conditions on $b_{r}$ and $b_{z}$. In three-dimensional simulations we use

$$
b_{r}=0 \quad \text { on horizontal boundaries; } \quad b_{z}=0 \quad \text { on vertical boundaries. }
$$

As explained in Ref. [15] these boundary conditions are not exact for an isolated device in a currentfree exterior, but they provide a sufficient approximation in the low magnetic Reynolds number regime (in all our simulations we have $\mathrm{Rm}=\sigma \mu_{0} U_{\max } R \ll 1$, where $U_{\max }$ is the maximal flow intensity). We finally discuss the boundary conditions for $\rho_{\mathrm{Li}}$. As in [14], we model the electrolytealloy interface as a surface source of $\mathrm{Li}$, and we introduce the notation

$$
s_{\mathrm{Li}}=-\frac{J \mathcal{M}_{\mathrm{Li}}}{n_{e} F} \quad \text { in units of } \mathrm{kg} \mathrm{s}^{-1} \mathrm{~m}^{-2}
$$


for the surface mass flux entering the alloy. Here $\mathcal{M}_{\mathrm{Li}}$ is the molar mass of lithium, $n_{e}=1$ is the number of charges carried by lithium ions and $F=96485 \mathrm{~s} \mathrm{~A} \mathrm{~mol}^{-1}$ is the Faraday constant. All the other boundaries are impermeable to lithium. In conclusion the boundary conditions for $\rho_{\mathrm{Li}}$ are as follows:

$$
\left.D \partial_{r} \rho_{\mathrm{Li}}\right|_{r=R}=0,\left.\quad D \partial_{z} \rho_{\mathrm{Li}}\right|_{z=0}=0,\left.\quad D \partial_{z} \rho_{\mathrm{Li}}\right|_{z=H}=s_{\mathrm{Li}} .
$$

As initial condition, we always suppose that the fluid is at rest and $\rho_{\mathrm{Li}}=\rho_{\mathrm{Li}, *}$ is uniform, and we use $\rho_{\mathrm{Li}, *}$ as reference state in the Boussinesq approximation. The initial conditions for the magnetic field are less important as the magnetic field almost immediately adjusts due to the high magnetic diffusion (we are close to the magnetostatic limit).

\section{B. SFEMaNS solver}

We solve the system of equations described in the previous section by using the numerical code SFEMaNS, developed since $2001[19,20]$. SFEMaNS participated to many international benchmarks on dynamo problems [21,22]. Motivated by the LMB topic, we have extended the code using a level-set method to be able to solve multiphase MHD problems [23]. This allowed us to study the Tayler instability [24], the metal pad roll instability [25], and the electrovortex flow mechanism [15] in multiphase simulations of LMBs. The present study mainly invokes the single-phase solver augmented with the advection-diffusion equation for $\rho_{\mathrm{Li}}$ and the buoyancy term. In Appendix B, we use the multiphase version of the code to study the hydrodynamical boundary condition at the alloy-salt interface. All the details on the numerical method used by SFEMaNS are given in the cited references, and for the purpose of this article it is sufficient to know that all the fields are spectrally decomposed on a Fourier basis along the azimuth and use a finite-element basis in the meridian plane. As an example, we expand the flow field as:

$$
\boldsymbol{u}=\sum_{m=0}^{M-1} \boldsymbol{u}_{m}^{c}(r, z, t) \cos (m \theta)+\sum_{m=1}^{M-1} \boldsymbol{u}_{m}^{s}(r, z, t) \sin (m \theta) .
$$

Here $\boldsymbol{u}_{m}^{c}(r, z, t)$ and $\boldsymbol{u}_{m}^{s}(r, z, t)$ are time-dependent piecewise quadratic finite-element functions and $m$ is the azimuthal wave number. The code is parallelized using domain decomposition in the meridian plane and along the different Fourier modes. A noticeable advantage of SFEMaNS is that axisymmetrical simulations $(M=1)$ can be done next to highly resolved, massively parallel, three-dimensional simulations. Table I provides numerical information on the simulations that have been done for this paper. For each of the figures shown in the article, we provide the maximal number of Fourier modes, $M$, the meridian mesh size, and the time step, $\Delta t$.

\section{Postprocessing the electrical potential of the battery}

Although we are mainly interested in the motion and in the composition of the alloy, it is of interest to postprocess some typical battery characteristics that are of interest to a wider community. We evaluate the surface-averaged molar fraction (at the interface $z=H$ with the salt) and volumeaveraged molar fraction as follows:

$$
\left\langle x_{\mathrm{Li}}\right\rangle_{S}=\left.\frac{1}{\pi R^{2}} \int_{S} x_{\mathrm{Li}}\right|_{z=H} d S, \quad\left\langle x_{\mathrm{Li}}\right\rangle_{V}=\frac{1}{\pi R^{2} H} \int_{V} x_{\mathrm{Li}} d V .
$$

The surface-averaged molar fraction allows us to calculate the potential of the cell. For this purpose we need to invoke the height of the electrolyte, $H_{e}$, and the conductivity of the molten salt composing the electrolyte, $\sigma_{e}$. In the paper we assume that the electrolyte layer is fairly thick: $H_{e}=1 \mathrm{~cm}$, and we use the realistic conductivity $\sigma_{e}=187 \mathrm{~S} \mathrm{~m}^{-1}$. Then we use the formula

$$
E_{\text {cell }} \approx \Phi\left(\left\langle x_{\mathrm{Li}}\right\rangle_{S}\right)+\frac{J H_{e}}{\sigma_{e}} \quad \text { with } \quad \Phi\left(x_{\mathrm{Li}}\right) \approx 0.614-0.598\left(x_{\mathrm{Li}}-0.17\right) \mathrm{V}
$$


TABLE I. Details on the numerical simulations discussed in this article. Referring to each figure, we provide the number of Fourier modes, $M$, the interval of (nonuniform) mesh sizes of the finite-element grid in the meridian plane. The time step $\Delta t$ is fixed for each simulation but changes from one simulation to the other. Intervals $\ldots \rightarrow \ldots$ indicate how $\Delta t$ was changed with increasing $J$.

\begin{tabular}{lccc}
\hline \hline Figures & $M$ & Mesh size $(\mathrm{mm})$ & $\Delta t(\mathrm{~s})$ \\
\hline 3 and 4 & 1 & {$[0.2,1]$ or $[0.1,0.2]$} & $5 \rightarrow 2 \times 10^{-3}$ \\
5 & 1 & {$[0.1,0.2]$} & $5 \times 10^{-4}$ \\
6 & 1 & {$[0.1,0.2]$} & $2 \times 10^{-2}$ \\
7 & 1 & {$[0.1,0.2]$} & $5 \times 10^{-2}$ \\
$8-10$ & 1 & {$[0.1,0.2]$} & $5 \times 10^{-2} \rightarrow 10^{-2}$ \\
9 & 1 & {$[0.1,0.2]$} & $5 \times 10^{-2}$ \\
11 & $1($ axi) & {$[0.1,0.2]$} & $10^{-3}$ \\
11 & $256(3 \mathrm{D})$ & {$[0.1,0.2]$} & $10^{-3}$ \\
12 & 1 & {$[0.1,0.2]$} & $10^{-3} \rightarrow 4 \times 10^{-5}$ \\
13 & 1 & {$[0.1,0.2]$} & $4 \times 10^{-5}$ \\
15 & 1 & {$[0.125,0.25]$} & $10^{-3}$ \\
\hline \hline
\end{tabular}

where, based on experimental data from Ref. [26], the open gate potential $\Phi$ is approximated by a linear fit valid over the entire range $x_{\mathrm{Li}} \in[17,60] \%$ (see Appendix A). The term $J H_{e} / \sigma_{e}$ is added to account for ohmic losses in the electrolyte layer.

When the alloy is not well mixed, the surface-averaged molar fraction near the interface with the alloy $($ at $z=H$ ) can be very different from the volume-averaged molar fraction [the problem illustrated in Fig. 1(b)]. This causes a so-called mass transfer overpotential which we calculate as

$$
\eta_{\mathrm{mt}}=\left|\Phi\left(\left\langle x_{\mathrm{Li}}\right\rangle_{V}\right)-\Phi\left(\left\langle x_{\mathrm{Li}}\right\rangle_{S}\right)\right| \text {. }
$$

$\eta_{\mathrm{mt}}$ measures the increase or decrease of electrical cell potential during charge or discharge due to local inhomogeneity in the alloy. It is a practical measure of how inhomogeneity in the alloy degrades the performance of the battery.

\section{Idealized, opposite limits to compare with: Perfect blend and pure diffusion}

We are going to compare the numerical simulations with two idealized and opposite limits. We assume in the first case that the alloy is instantaneously perfectly blended and we assume in the second case that mixing is done only by molecular diffusion. These two extreme situations are illustrated in Fig. 2.

In the first case the alloy is perfectly blended but we entirely ignore how this is done (for example, by vigorous turbulent flow). We assume that all the Li that enters or leaves the alloy is immediately redistributed throughout the entire volume of the alloy, see Fig. 2(a). The almost homogeneous density field then evolves in time as follows:

$$
\text { (best) } \quad \rho_{\mathrm{Li}}(t)=\rho_{\mathrm{Li}, *}+\frac{s_{\mathrm{Li}} t}{H} \text {. }
$$

Here $\rho_{\mathrm{Li}, *}$ is the initial reference density of $\mathrm{Li}$ in the alloy. In this limit, there is no mass-transfer overpotential, $\eta_{\mathrm{mt}}=0 \mathrm{~V}$, and intermetallics only form at the last moment when the molar fraction $x_{\mathrm{Li}}$ exceeds $60 \%$ everywhere. This is clearly the best-case scenario.

In the second case, there is only molecular diffusion to transport $\mathrm{Li}$ in the $\mathrm{Li}-\mathrm{Pb}$ alloy. This is the worst-case scenario. Considering that molecular diffusion is very weak, this is utterly inefficient and it results in a very large inhomogeneity level in the alloy occurring right under the electrolyte-alloy 


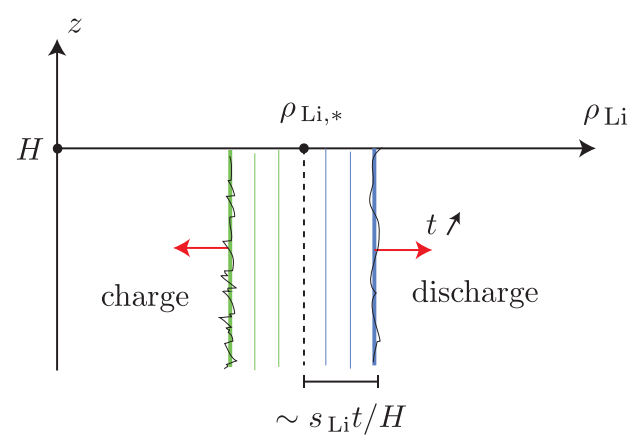

(a) perfect blend

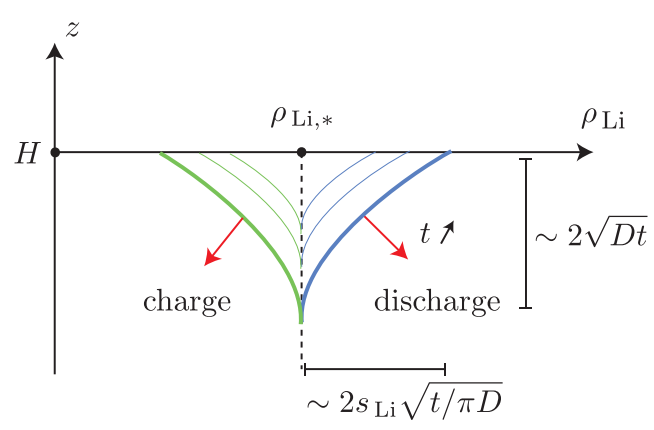

(b) pure diffusion

FIG. 2. Sketch of idealized scenarios for the time evolution of $\rho_{\mathrm{Li}}$ in the alloy. (a) Best-case scenario with a perfectly blended alloy: With a very efficient mixing process, the alloy is almost homogeneous at all times and $\rho_{\mathrm{Li}}$ closely follows the volume average $\left\langle\rho_{\mathrm{Li}}\right\rangle_{V} \sim s_{\mathrm{Li}} t / H$. (b) Worst-case scenario with only diffusion: The distance traveled by the diffusive front into the fluid at time $t$ is $2 \sqrt{D t}$, the concentration difference between the bulk, and the interface is $2 s_{\mathrm{Li}} \sqrt{t / \pi D}$.

interface. From Ref. [27, p. 75 and p. 112] and Ref. [14], we know the exact analytical solution for the purely diffusive problem:

$$
\text { (worst) } \begin{aligned}
\rho_{\mathrm{Li}}(z, t)= & \rho_{\mathrm{Li}, *}+\frac{s_{\mathrm{Li}} t}{H}+\frac{s_{\mathrm{Li}} H}{D}\left[\frac{3 z^{2}-H^{2}}{6 H^{2}}-\frac{2}{\pi^{2}} \sum_{n=1}^{+\infty} \frac{(-1)^{n}}{n^{2}}\right. \\
& \left.\times \exp \left(\frac{-n^{2} \pi^{2} D t}{H^{2}}\right) \cos \left(\frac{n \pi z}{H}\right)\right] .
\end{aligned}
$$

We are going to compare systematically the numerical simulations with this solution in all the graphs reported below. We have verified that SFEMaNS correctly reproduces this diffusive solution in absence of flow, i.e., with $\boldsymbol{u}=\mathbf{0}$. In some theoretical arguments, we will use the semifinite approximation (bottom lid at $z \rightarrow-\infty$ ), valid for $t \ll H^{2} / D$ :

$$
\text { (worst, semi- } \infty) \quad \rho_{\mathrm{Li}}(z, t) \approx \rho_{\mathrm{Li}, *}+2 s_{\mathrm{Li}} \sqrt{\frac{t}{D}} \operatorname{ierfc}\left(\frac{H-z}{2 \sqrt{D t}}\right) .
$$

Here $\operatorname{ierfc}(x)=\frac{1}{\sqrt{\pi}} \exp \left(-x^{2}\right)-x \operatorname{erfc}(x)$ and $\operatorname{erfc}(x)$ is the complementary error function. We illustrate the main properties of the diffusive solution in Fig. 2(b). Since the distance traveled by the diffusive front into the fluid at time $t$ is $2 \sqrt{D t}$, the concentration difference between the bulk and the interface is $2 s_{\mathrm{Li}} \sqrt{t / \pi D}$.

\section{DISCHARGING CELLS}

\section{A. Simulations}

We first consider the case of a discharging cell. The alloy is initially homogeneous and we set $x_{\mathrm{Li}}=17 \%$. A constant mass flux of light Li uniformly leaves the electrolyte and enters the positive electrode at the top and diffuses into the alloy. This locally increases $x_{\mathrm{Li}}$ and makes the alloy lighter and stably stratified near the interface with the electrolyte.

Due to the thinner shape of the wire electrode at the bottom, the electrovortex mechanism generates a flow that pushes the bulk alloy toward the inhomogeneous top layer. Let us introduce the nondimensional number $S=\mu_{0} J^{2} R^{4} / 4 \rho v^{2}$. The magnitude of the electrovortex flow increases with $S$. It behaves like $S$ in the Stokes regime (i.e., when $S$ is small) and like $\sqrt{S}$ in the inertial regime (i.e., when $S$ is large) [28]. In this section we vary $S=\left\{10,10^{3}, 4 \times 10^{3}, 10^{5}\right\}$ to explore 


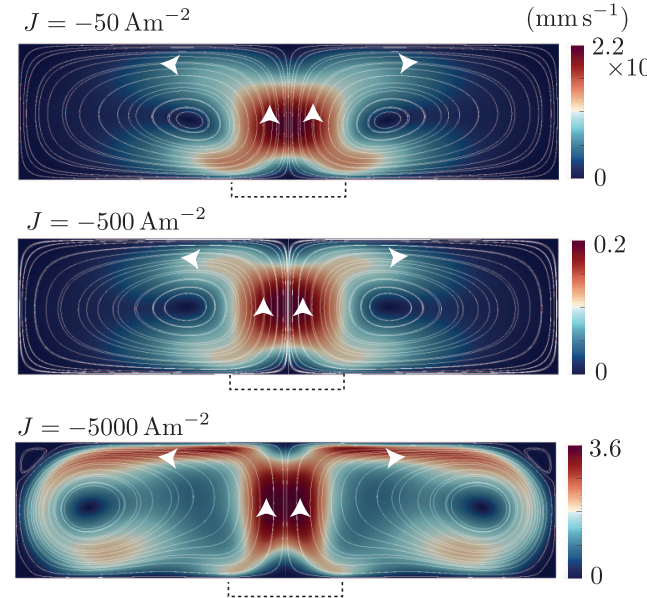

(a) $\|\boldsymbol{u}\|$
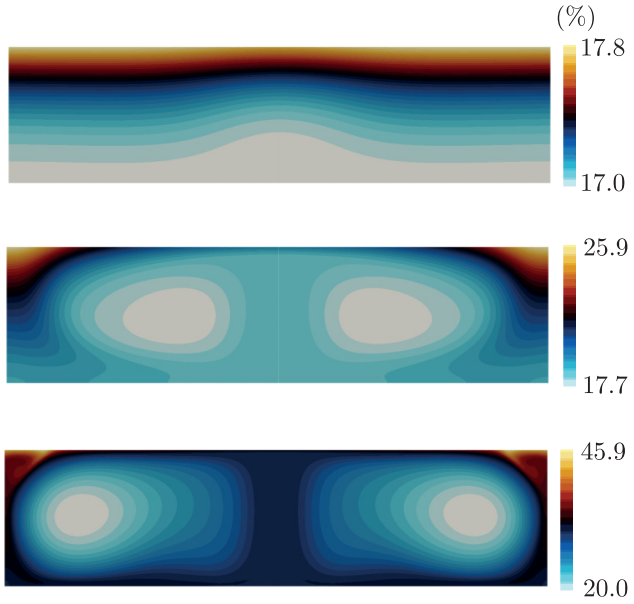

(b) $x_{\mathrm{Li}}$

FIG. 3. Discharging cells without buoyancy. Snapshots in the meridian section of the magnitude of the velocity field $\|\boldsymbol{u}\|$ (left panels) and of the molar fraction $x_{\mathrm{Li}}$ (right panels) for varying current densities $J$ and at times $t=5556 \mathrm{~s}$ (top), $t=6111 \mathrm{~s}$ (middle), and $t=2889 \mathrm{~s}$ (bottom). The electrovortex flow effectively homogenizes the alloy in the bulk when $J$ is large, but this mechanism does not mix well the alloy: Large concentrations always build up in the upper left and right corner of the cavity [near $(r, z)=(R, H)]$.

both the low and the high $S$ regimes. In the small cell considered in this paper (recall that $R=4 \mathrm{~cm}$ and $H=2 \mathrm{~cm}$ ), this corresponds to realistic values $J \in\{-50,-500,-1000,-5000\} \mathrm{A} \mathrm{m}^{-2}$. Our objective is to investigate how the electrovortex flow affects the composition of the alloy phase. All the simulations reported here are axisymmetric. This is not a limitation considering the results of Ref. [15].

\section{Without solutal buoyancy}

To appreciate the role of solutal buoyancy, we first present simulations without buoyancy. This is done in SFEMaNS by setting $g=0$. The concentration field $\rho_{\mathrm{Li}}$ evolves in time as if it were a passive scalar transported by the flow induced by the electrovortex mechanism.

In Fig. 3, we show typical snapshots in the meridian section of the velocity field $\boldsymbol{u}$ and of the molar fraction $x_{\mathrm{Li}}$ at some late times for the current densities $J \in\{-50,500,5000\} \mathrm{A} \mathrm{m}^{-2}$. The figures are on-scale and the dashed lines in Fig. 3(a) represent the copper wire connected to the cell at its bottom. For the lowest current density, $J=-50 \mathrm{~A} \mathrm{~m}^{-2}$ the cell operates in the Stokes regime (or low-S regime). A creeping flow of only a few $\mu \mathrm{m} \mathrm{s}^{-1}$ is present. It is oriented upward near the axis. Such a weak flow can only slightly modify the $x_{\mathrm{Li}}$ distribution, which is here nearly in the purely diffusive state. As the current density increases from $J=-500 \mathrm{~A} \mathrm{~m}^{-2}$ to $-5000 \mathrm{~A} \mathrm{~m}^{-2}$ the electrovortex flow intensifies and the flow magnitude reaches a few $\mathrm{mm} \mathrm{s}^{-1}$ in the cell. Without solutal buoyancy, the molar fraction distribution $x_{\mathrm{Li}}$ is greatly influenced by the electrovortex flow. The bulk becomes more homogeneous, but we also observe that there is still an accumulation of $\mathrm{Li}$ near the stagnation points in the upper left and right corners of the cell. These simulations show that in the cell under consideration, the electrovortex flow mixes the alloy but it does not do it very well.

Figure 4 quantitatively compares the results from the numerical simulations to the theoretical purely diffusive case (dashed lines) for the currents $J \in\{-50,500,5000\} \mathrm{Am}^{-2}$. Recall that the diffusive solution represents the worst-case scenario. As shown in Fig. 4(a), the difference $\Delta x_{\mathrm{Li}}=$ $\max \left(x_{\mathrm{Li}}\right)-\min \left(x_{\mathrm{Li}}\right)$, which measures the inhomogeneity level of the alloy, inevitably increases in time both for the theoretical diffusive solution and the numerical simulations, but it increases less rapidly in the numerical simulations where the electrovortex flow is active. The mixing induced 


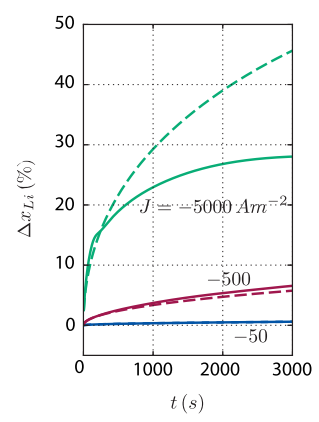

(a) inhomogeneity

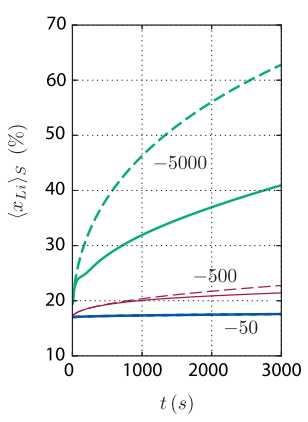

(b) surface molar fraction

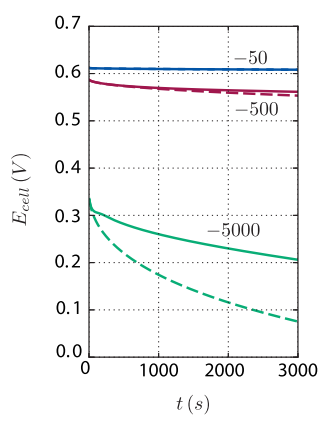

(c) cell voltage

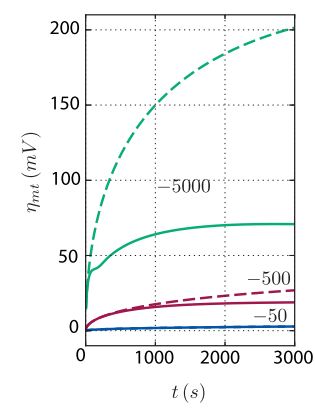

(d) overpotential

FIG. 4. Discharging cell without buoyancy. The purely diffusive analytical solution (dashed lines) is compared to the numerical simulations (full lines) for varying current densities $J$, as marked in the figure. (a) Inhomogenity in the cell measured by $\Delta x_{\mathrm{Li}}=\max \left(x_{\mathrm{Li}}\right)-\min \left(x_{\mathrm{Li}}\right)$. (b) Average composition at the interface with the electrolyte. (c) Theoretical cell voltage. (d) Mass transfer overpotential. The flow in the alloy homogenizes the molar fraction and lowers the concentration at the interface. As a result the cell voltage drops less quickly than in the purely diffusive regime; in other words, the electrovortex flow reduces the mass transfer overpotential.

by the electrovortex flow has also an impact on the average surface concentration as observed in Fig. 4(b). It is clearly lower when the electrovortex flow is active. From $\left\langle x_{\mathrm{Li}}\right\rangle_{S}$ and (13) we deduce the time evolution of the cell's potential, $E_{\text {cell }}$, and the results are shown in Fig. 4(c). The internal resistance, $J H_{e} / \sigma_{e}$, has a large impact on the value of $E_{\text {cell }}$ at $t=0$ because we somewhat arbitrarily choose the large value $H_{e} \approx 1 \mathrm{~cm}$, but other values for $H_{e}$ can be used in (13). The piece of information that is important in Fig. 4(c) is the decrease in time of the potential. We show in Fig. 4(d) the mass transfer overpotential $\eta_{\mathrm{mt}}$. This plot demonstrates a little better than the previous one that, if buoyancy were indeed negligible, the electrovortex flow would have a net positive influence on the cell's potential and would thereby increase the efficiency of the cell.

\section{With solutal buoyancy}

We now take buoyancy into account in SFEMaNS by using a realistic gravity coefficient $g=$ $9.81 \mathrm{~m} \mathrm{~s}^{-2}$. We thus obtain a better representation of reality in which the electrovortex flow needs to counter the stabilizing effects of the solutal buoyancy.

In Fig. 5, we show three snapshots of the molar fraction $x_{\mathrm{Li}}$ and of the streamlines of the velocity field at different times and at the high discharge rate $J=-5000 \mathrm{~A} \mathrm{~m}^{-2}$. The electrovortex flow is intense at the beginning, but it does not deem to be strong enough to penetrate the stagnant layer of lighter, Li-enriched alloy, floating at the top of the electrode. As time increases, the thickness of the stagnant layer grows under the action of diffusion and the electrovortex flow gradually vanishes as indicated by the numerical values of $\max (\|\boldsymbol{u}\|)$ reported in the figure captions. This series of simulations shows that in some parts of the electrode the Lorentz force $\boldsymbol{j} \times \boldsymbol{b}$ can be balanced and even be overcome by the buoyancy force.

We have also observed this behavior for lower discharge currents, $J=-50,-500 \mathrm{~A} \mathrm{~m}^{-2}$ (not reported here). We conclude then that, in the LMB configuration under consideration and for realistic current densities, the electrovortex flow mechanism cannot prevent the formation of a stagnant buoyant layer at the interface with the electrolyte. Inside this stagnant layer, the composition of the alloy is entirely controlled by the diffusion process and the alloy is just as inhomogeneous as it would be without the action of the electrovortex flow. This observation also means that the dashed lines in Fig. 4, which represent the worst-case scenario, i.e., the diffusive regime, are actually the realistic ones. This claim is supported by the numerical simulations which we do not report for brevity. 


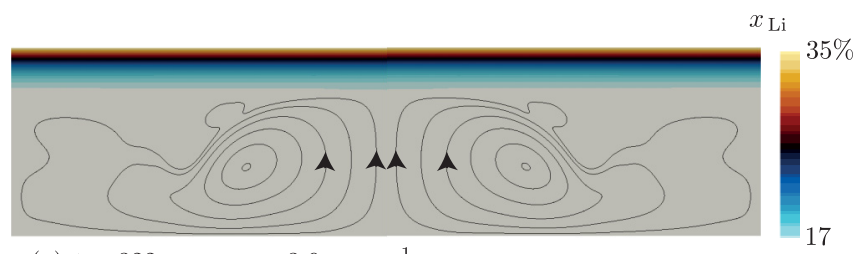

(a) $t=322 \mathrm{~s}, u_{\max }=3.9 \mathrm{~mm} \mathrm{~s}^{-1}$

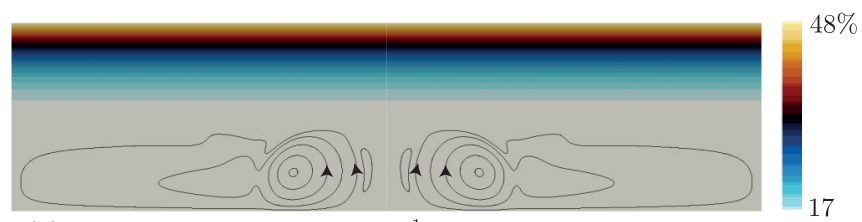

(b) $t=1167 \mathrm{~s}, u_{\max }=3.0 \mathrm{~mm} \mathrm{~s}^{-1}$

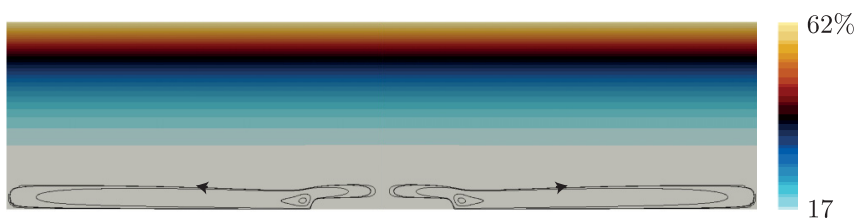

(c) $t=2944 \mathrm{~s}, u_{\max }=0.2 \mathrm{~mm} \mathrm{~s}^{-1}$

FIG. 5. Discharging cell with buoyancy. Snapshots of the velocity streamlines and of the molar fraction $x_{\mathrm{Li}}$ for the current density $J=-5000 \mathrm{~A} \mathrm{~m}^{-2}$ at times: $t=322 \mathrm{~s}$ (top); $t=1167 \mathrm{~s}$ (middle); $t=2944 \mathrm{~s}$ (bottom). The electrovortex flow is active in the alloy but never penetrates the stagnant and stably stratified layer that is created near the upper interface during discharge. At late times the electrovortex flow almost entirely vanishes, as indicated by the numerical values of $u_{\max }$.

\section{B. Physical analysis of mixing during discharge}

In the preceding section we have observed two extreme scenarios. When buoyancy is inactive the electrovortex flow mixes the alloy in the entire electrode. When buoyancy is active the intensity of the electrovortex flow is not strong enough to penetrate the stable layer floating at the top of the electrode, and the molar fraction $x_{\mathrm{Li}}$ close to the interface with the electrolyte is completely controlled by diffusion. The question we want to investigate now is how intense should a generic fluid flow be to be able to counteract the stabilizing effects of buoyancy? In the following sections, using simple physical arguments, we define two characteristic velocity scales, $U_{p}$ and $U_{m}$, that answer this question. We test the relevance of these velocity scales by running numerical simulations with artificially low values of the gravity coefficient and observe the mixing properties of the electrovortex flow.

\section{Minimal flow magnitude $U_{p}$ to pierce through the stratified layer}

The fluid being initially at rest, it is unavoidable that at least an advection time unit, $H / U$, goes by for a fluid flow with intensity $U$ to develop in the cell. During this time, a stably stratified layer will have been created and a minimal flow intensity denoted $U_{p}$ will be necessary to pierce through this layer.

To estimate the characteristic velocity $U_{p}$, we start from the diffusive solution (16b) which is a precise approximation for $\rho_{\mathrm{Li}}$ for short advection times. Using (3a) and (16b), we obtain the following profile for the difference in density between the bulk alloy and the light alloy at the top:

$$
\Delta \rho(z, t)=\rho_{*}-\rho(z, t)=2 \chi s_{\mathrm{Li}} \sqrt{\frac{t}{D}} \operatorname{ierfc}\left(\frac{H-z}{2 \sqrt{D t}}\right) .
$$


A parcel of the heavier bulk fluid can rise upward through the stratification and reach the upper boundary only if the bulk flow provides the necessary work to act against gravity. We conjecture that the energy density balance

$$
\frac{\rho_{*} U(t)^{2}}{2} \approx \int_{z \text { bulk }}^{H} \Delta \rho(z, t) g d z
$$

expresses this physical idea. Here $U(t)$ is the typical flow magnitude that is needed to pierce through the stably stratified layer that formed at time $t$. A similar argument was used in $[15,24]$ to estimate the interface elevation caused by a fluid flow in a multiphase modeling of a LMB. The coordinate $z_{\text {bulk }}$ is that of an arbitrary point in the nonstratified bulk fluid. Since $\Delta \rho\left(z_{\text {bulk }}, t\right)$ goes exponentially fast to zero when $H-z$ is larger that $2 \sqrt{D t}$, the integral is very well approximated by the asymptotic value in the limit $z_{\text {bulk }} \rightarrow-\infty$. Changing variables $s=(H-z) / 2 \sqrt{D t}$ in the integral and using the analytical result $\int_{0}^{+\infty} \operatorname{ierfc}(s) d s=1 / 4$, we simplify (18) to

$$
\frac{U(t)^{2}}{2} \approx s_{\mathrm{Li}} \beta g t,
$$

where we recall that $\beta=\chi / \rho_{*}$ is the solutal expansion coefficient. We now express that a flow of magnitude $U$ needs at least an advection timescale $t=H / U$ to develop. Then we define $U_{p}$ to be the solution of the equation $U\left(H / U_{p}\right)=U_{p}$. Replacing $s_{\mathrm{Li}}$ in (19) by its definition (9) and reorganizing the terms, we find

$$
U_{p}=\left(\frac{2|J| \mathcal{M}_{\mathrm{Li}} \beta g H}{n_{e} F}\right)^{1 / 3} .
$$

The quantity $U_{p}$ is the flow intensity that is required to pierce the stratified layer that is formed at time $t=H / U_{p}$. Since the stratification grows in time, a flow with intensity $U \ll U_{p}$ is unlikely capable of piercing through the stratified layer at any time. Therefore we posit that $U \gg U_{p}$ is a minimal condition for mixing.

We now test the relevance of the characteristic velocity $U_{p}$ by running some simulations under microgravity with $J=-1 \mathrm{kA} \mathrm{m}^{-2}$. We start by setting $g=0$, and we measure the maximal speed in the electrovortex flow. We obtain $U_{0}=0.55 \mathrm{~mm} \mathrm{~s}^{-1}$. From (20), we compute the critical gravity intensity $g_{p}=3.65 \times 10^{-2} \mathrm{~ms}^{-2}$ by solving $U_{p}\left(g_{p}\right)=U_{0}$. Then we run three simulations with microgravity $g \in\left\{0.1 g_{p}, g_{p}, 10 g_{p}\right\}$ and observe the effects of the electrovortex flow. In Fig. 6, we show the time evolution of the molar fraction in half of the meridian section for various times. For $g \gg g_{p}$ the stratification is mostly unaffected, for $g \ll g_{p}$ the flow significantly deforms the stratification. For $g=g_{p}$ the intensity of the flow is large enough to pierce through the stratified layer near the center of the cell and for a short amount of time. The organization of the flow also depends on $g$ : it spreads out in the radial direction when gravity is low, as suggested by the dashed lines in the bottom panels in the figure. Overall, this test suggests that the criterion $U \approx U_{p}$ correctly estimates the intensity of the flow that is needed to pierce through the stratified layer.

Applied to the small battery under study, $J=(-50,-500,-5000) A m^{-2}$, and with the real value of the gravity coefficient $g=9.81 \mathrm{~m} \mathrm{~s}^{-2}$, we obtain the characteristic flow speed $U_{p}=$ $(1.3,2.8,6.1) \mathrm{mm} \mathrm{s}^{-1}$, respectively. The typical flow magnitude measured in the numerical simulations with $g=0$ and the same current densities $J=(-50,-500,-5000) \mathrm{Am}^{-2}$ are $U=$ $\left(2.2 \times 10^{-3}, 0.2,3.6\right) \mathrm{mm} \mathrm{s}^{-1}$, respectively [see Fig. 3(a)]. In the three cases, we have $U<U_{p}$ which explains why the electrovortex flow is indeed too weak to influence mixing.

\section{Minimal flow magnitude $U_{m}$ for efficient mixing}

We now introduce a second characteristic velocity $U_{m}$ larger than $U_{p}$. Rather than just demanding that the bulk flow be able to pierce through the stratification, we demand that the flow should be sufficiently intense to transport lighter fluid parcels located close to the interface with the electrolyte into the bulk. Given some velocity scale $U$ of the flow, we start by evaluating the maximal density 


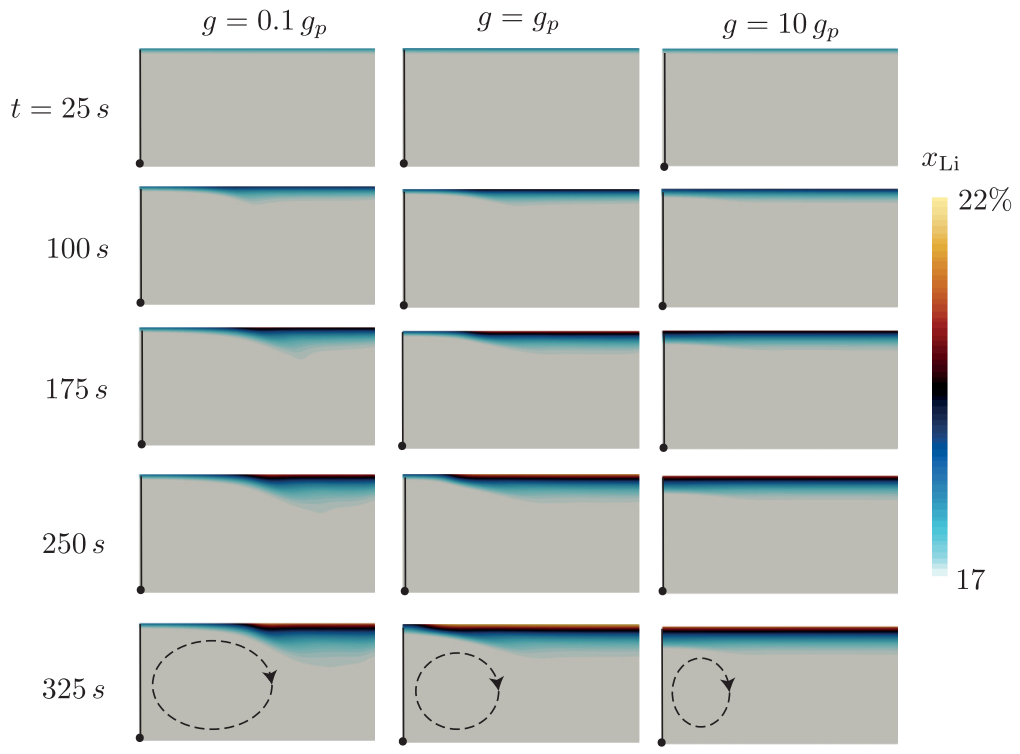

FIG. 6. Discharging cell with low buoyancy. Below the flow intensity $U_{p}$, see (20), the bulk flow cannot pierce the stratified layer and therefore cannot mix the alloy close to the interface with the electrolyte. We test this criterion under microgravity. With $J=-1 \mathrm{kA} \mathrm{m}^{-2}$, the magnitude of the velocity in the electrovortex flow reaches $U_{p}$ if $g=g_{p}=3.65 \times 10^{-2} \mathrm{~m} \mathrm{~s}^{-2}$. Here we show the molar fraction in half of the meridian section as a function of time for $g \in\left\{0.1 g_{p}, g_{p}, 10 g_{p}\right\}$, (the vertical axis and the coordinate origin are represented by a black line and a dot). For $g=0.1 g_{p}$, the flow pierces the buoyancy layer. For $g=10 g_{p}$ the flow never penetrates the buoyancy layer.

difference between the bulk and the top of the electrode at the time $t=H / U$. Using that ierfc $(0)=$ $1 / \sqrt{\pi}$, the diffusion solution (17) gives

$$
\Delta \rho_{\mathrm{adv}}(U)=2 \chi s_{\mathrm{Li}} \sqrt{\frac{H}{\pi U D}} .
$$

Moving the light fluid parcels from where they are created, $z=H$, to the bottom of the electrode, $z=0$, requires an amount of work of about $\Delta \rho_{\text {adv }} g H$ per unit volume. We posit that this amount of energy should be retrieved from the available kinetic energy in the bulk of the flow. We express this through the balance

$$
\frac{\rho_{*} U^{2}}{2}=\Delta \rho_{\mathrm{adv}}(U) g H
$$

We then define $U_{m}$ to be the solution of the equation $\frac{\rho_{*} U_{m}^{2}}{2}=\Delta \rho_{\mathrm{adv}}\left(U_{m}\right) g H$. Replacing $s_{\mathrm{Li}}$ by its definition, we find

$$
U_{m}=\left(\frac{4 \beta|J| \mathcal{M}_{\mathrm{Li}} g}{n_{e} F}\right)^{2 / 5} \frac{H^{3 / 5}}{(\pi D)^{1 / 5}} .
$$

Any fluid flow with a velocity scale $U$ such that $U \gg U_{m}$ should be able to transport light fluid all around the cell, and hence should be less influenced by solutal buoyancy. We conclude that a flow should have a good mixing capacity if $U \gg U_{m}$, but of course the quality of the mixing still depends on the topology of the flow.

We study the significance of the second velocity scale $U_{m}$ by running a second series of numerical simulations with a small gravity coefficient and with $J=-1 \mathrm{kA} \mathrm{m}^{-2}$. Recalling that the maximum 

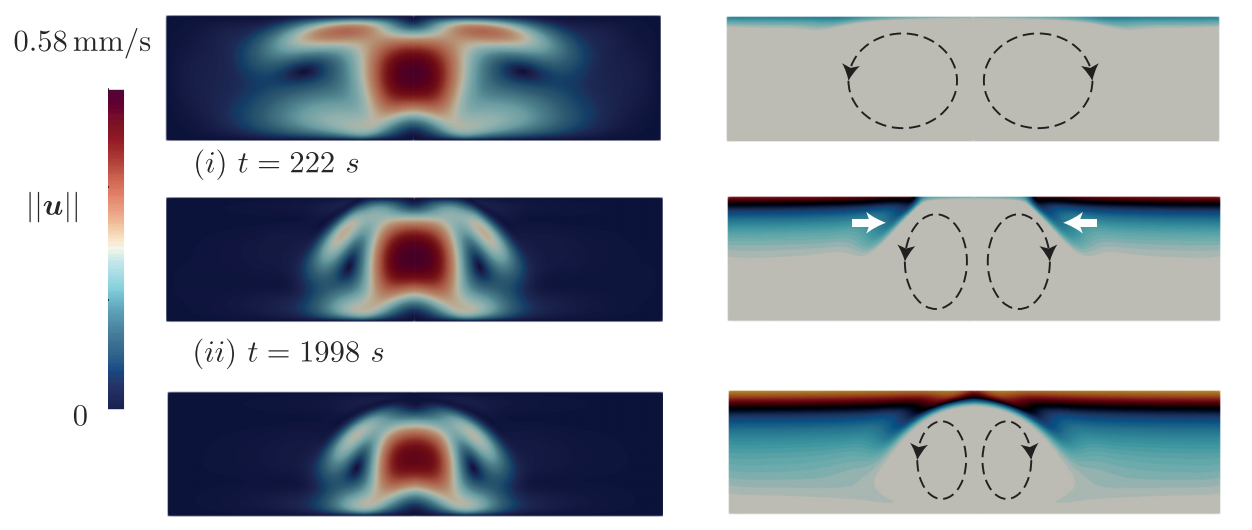

$30 \%$

(iii) $t=3774 \mathrm{~s}$

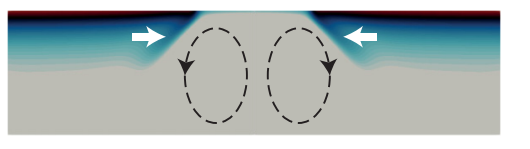

$x_{\mathrm{Li}}$

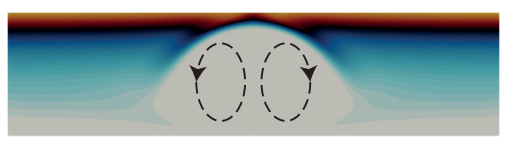

17

FIG. 7. Discharging cell with low buoyancy. Snapshots of the velocity and of the molar fraction at times $t=222 \mathrm{~s}$ (top), $t=1998 \mathrm{~s}$, and $t=3774 \mathrm{~s}$ with the low gravity coefficient $g=1.7 \times 10^{-3} \mathrm{~m} \mathrm{~s}^{-2}$ and current density $J=-1 \mathrm{kA} \mathrm{m}^{-2}$. The flow pierces through the stratification and influences the composition of the alloy for a significant amount of time but ultimately gets disconnected as the stratification intensified.

velocity in the electrovortex flow is $U_{0}=0.55 \mathrm{~mm} \mathrm{~s}^{-1}$ with this current density when $g=0$, we compute the gravity intensity $g_{m}$ so that $U_{m}\left(g_{m}\right)=U_{0}$. We obtain $g_{m}=8.7 \times 10^{-4} \mathrm{~m} \mathrm{~s}^{-2}$, which is a much lower value than $g_{p}$.

Let us now observe what happens slightly above the critical value $g_{m}$. In Fig. 7 we show snapshots of the flow intensity and of the molar fraction with the gravity coefficient $g=2 g_{m}=$ $1.7 \times 10^{-3} \mathrm{~m} \mathrm{~s}^{-2}$ and $J=-1 \mathrm{kAm}^{-2}$. In snapshot (i), we observe that, after the cell starts discharging, the electrovortex flow clearly pierces through the stratified layer and blows fresh alloy more or less directly onto the interface. The flow obviously influences the composition of the alloy, since it pushes radially the light alloy formed at the interface and replaces it by new material. In snapshot (ii), we see that as time passes the light material accumulates in the corners and creates a front of stratified stagnant fluid that thickens. This front slowly moves radially inward as suggested by the pair of thick white arrows. In snapshot (iii), we observe that at later times the diffusion front ultimately recaptures control of the center of the cell. After this time, the situation does not evolve very much anymore. The flow significantly deforms the stratification over the entire height of the cell, but it no longer reaches the interface with the electrolyte and so its capacity of moving away the light material that further enters the cell is limited. Notice that the process described above is analogous to the cylindrical hydraulic jump that forms in a pan that slowly fills up with tap water. There, too, the radius of the hydraulic jump decreases as time passes and the hydraulic jump eventually vanishes when the pan has filled up to a certain height.

To further our understanding of the influence of solutal buoyancy, we now explore the entire range of the gravity coefficient, $g \in[0,9.81] \mathrm{m} \mathrm{s}^{-2}$. In the left panel of Fig. 8, we show the time evolution of the root-mean-square velocity, $U_{\mathrm{rms}}$, in the cell while varying $g$ from 0 to $9.81 \mathrm{~m} \mathrm{~s}^{-2}$. Initially $U_{\text {rms }}$ increases and all the curves follow the same trend, but we observe that at later times the magnitude of the velocity decreases and the larger $g$ the faster $U_{\text {rms }}$ vanishes. In the right panel of Fig. 8, we show snapshots of the molar fraction at the final time $t=4444 \mathrm{~s}$. This series of images clearly shows the negative impact of solutal buoyancy as the gravity increases. Notice that these plots suggest that our theoretical estimate $g_{m}=8.7 \times 10^{-4}$ (dashed line) marks rather well the transition below which the bulk flow can reach the upper boundary $\left(g<g_{m}\right)$. As we have seen above, taking $g=2 g_{m}$ is already too large a value.

The system should be close to steady state after a few diffusive time units, which we estimate to be $H^{2} /\left(\pi^{2} D\right) \approx 5000 \mathrm{~s}$ from $(16 \mathrm{a})$. Hence the final computational time reached in the snapshots 


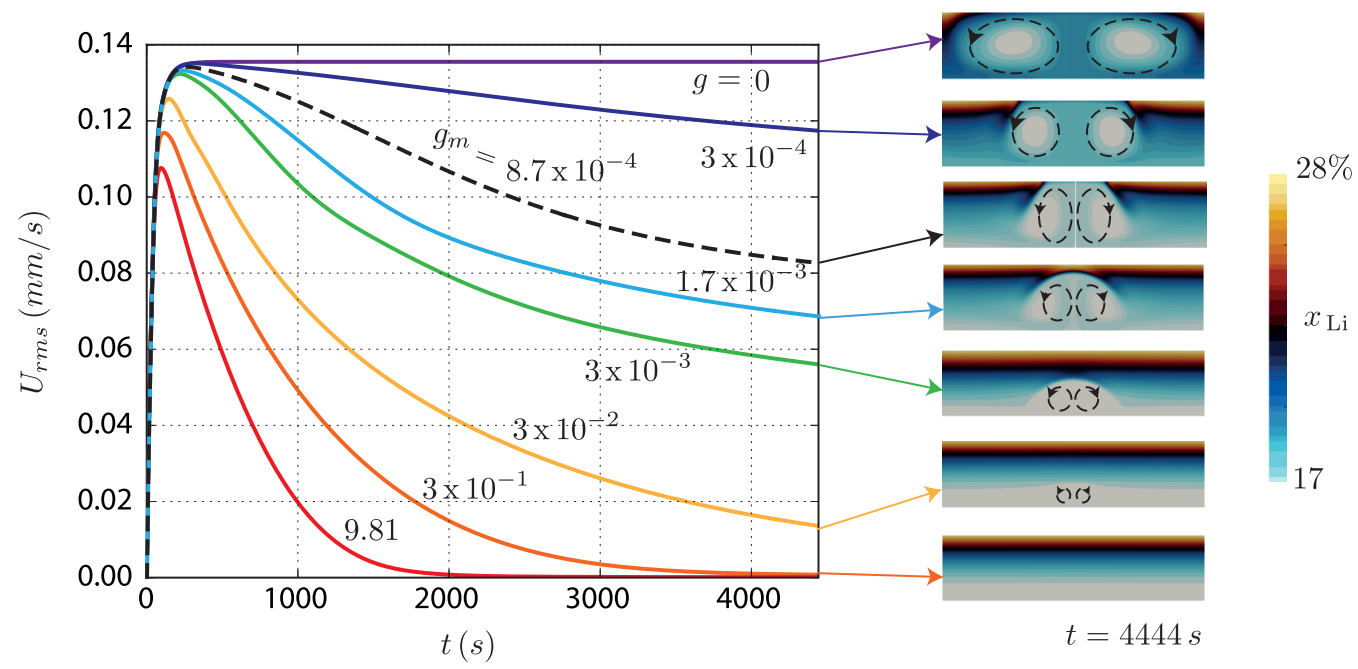

FIG. 8. Discharging cell with low buoyancy. Root-mean-square speeds as a function of time and snapshots of the molar fraction $x_{\mathrm{Li}}$ in simulations with decreasing gravitational accelerations $g$, as marked in the figure. The time evolution of rms speeds suggests that the electrovortex flow decreases over time. All the snapshots are taken at the fixed time $t=4444 \mathrm{~s}$ and using the fixed discharge current density $J=-1 \mathrm{kA} \mathrm{m}^{-2}$. For $g<g_{m}$ $\left[U>U_{m}\right.$, see (23)], the flow pierces through the stratification even at late time.

shown in Fig. 8 is not large enough to be close to steady state. In Fig. 9, we show snapshots of the molar fraction in the meridian section at the larger time $t=26600 \mathrm{~s}$ and for the following values of the gravity coefficient $g=3 \times 10^{-3}, 8.7 \times 10^{-4}, 3 \times 10^{-4} \mathrm{~m} \mathrm{~s}^{-2}$, which are close to $g_{m}$. This figure further confirms that the criterion $U>U_{m}$ (or equivalently $g<g_{m}$ ) seems to be a necessary condition on the intensity of the flow field to be able to pierce through the stratification at all times. This observation is a little bit surprising, considering that no long time asymptotic behavior has not been accounted for in the derivation of $U_{m}$. Nevertheless, it seems reasonable to think that if $U>U_{m}$ (or equivalently $g<g_{m}$ ), the flow is likely to mix better the alloy, as it can reach the interface with the electrolyte at all times.

Let us investigate the mixing capability of the electrovortex flow when $g$ varies. We show in Fig. 10 the time evolution of the maximal inhomogeneity criteria $\Delta \rho_{\mathrm{Li}}=\max \left(\rho_{\mathrm{Li}}\right)-\min \left(\rho_{\mathrm{Li}}\right)$ and $\Delta x_{\mathrm{Li}}=\max \left(x_{\mathrm{Li}}\right)-\min \left(x_{\mathrm{Li}}\right)$ over six diffusion time units for various values of the gravity coefficient. Whether the gravity is large or not, we see that the electrovortex flow does not mix well the alloy since the maximal inhomogeneity is always relatively large. Notice that $\Delta \rho_{\mathrm{Li}}$ reaches an
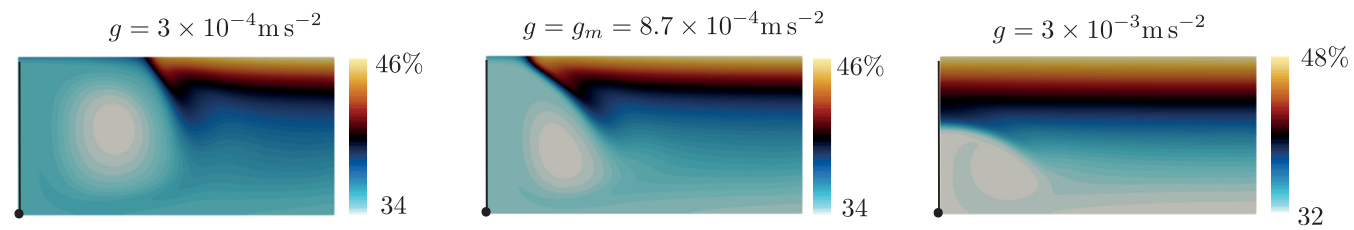

FIG. 9. Discharging cell with low buoyancy. Snapshots of the molar fraction in the meridian section at $t=$ $26600 \mathrm{~s}$ with $g \in\left\{3 \times 10^{-4}, 8.7 \times 10^{-4}, 3 \times 10^{-3}\right\} \mathrm{m} \mathrm{s}^{-2}$ and $J=-1 \mathrm{kAm}^{-2}$. We observe that $g \approx g_{m}$ or $[U \approx$ $U_{m}$, see (23)] is quite precisely the theshold below which the gravity is small enough to allow the electrovortex flow to pierce through the stratification at all times. Hence the flow influences the composition significantly if $U>U_{m}$. 


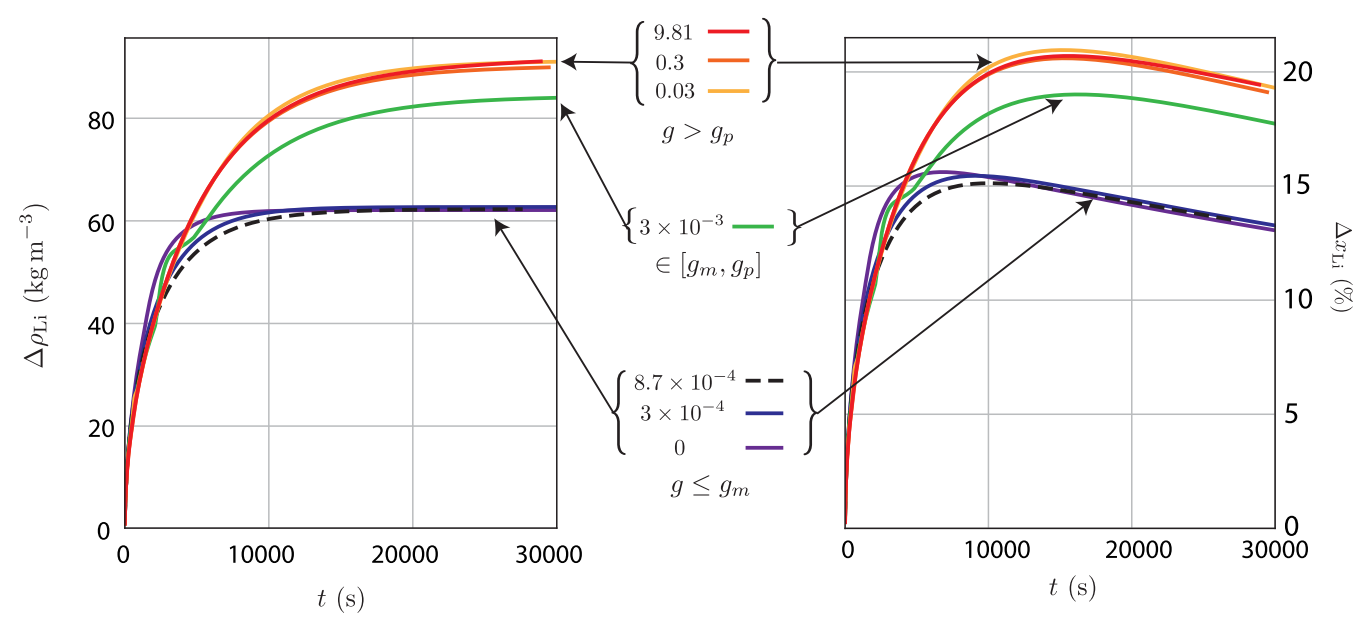

FIG. 10. Discharging cell with low buoyancy. Time evolution of the maximal inhomogeneity criteria in the alloy $\Delta \rho_{\mathrm{Li}}=\max \left(\rho_{\mathrm{Li}}\right)-\min \left(\rho_{\mathrm{Li}}\right)$ and $\Delta x_{\mathrm{Li}}=\max \left(x_{\mathrm{Li}}\right)-\min \left(x_{\mathrm{Li}}\right)$ with $g \in$ $\left\{0,0.0003, g_{m}, 0.003,0.03,0.3,9.81\right\} \mathrm{ms}^{-2}$ and $J=-1 \mathrm{kA} \mathrm{m}^{-2}$. When $g \gtrsim g_{p}$ (or equivalently $U \lesssim U_{p}$ ), the curves cluster around the purely diffusive solution. When $g<g_{m}$ (or equivalently $U>U_{m}$ ), the curves cluster around the no-gravity solution where mixing is maximal. When $g \in\left[g_{m}, g_{p}\right]$ (or equivalently $U \in\left[U_{p}, U_{m}\right]$ ), the mixing properties of the flow are moderate.

asymptote at large times, but $\Delta x_{\mathrm{Li}}$ does not. This is just a consequence of the definition of molar fraction. The most interesting aspect in these two graphes is that two clear branches emerge at long times. For the small values of the gravity coefficient $g \in\left\{0,3 \times 10^{-1}, 8.7 \times 10^{-4}\right\} \mathrm{ms}^{-2}$, i.e., $g \leqslant g_{m}$, we observe that the curves cluster around the no-gravity solution for which mixing is maximal. For the large values of the gravity coefficient $g \in\{0.03,0.3,9.81\}$, i.e., $g \gtrsim g_{p}$, the curves cluster around the purely diffusive solution where no mixing occurs. For $g=0.003 \mathrm{~m} \mathrm{~s}^{-2} \in\left[g_{m}, g_{p}\right]$ the mixing properties of the flow are moderate.

\section{Three regimes of mixing}

The results of the numerical simulations shown in the previous sections suggest that the quantities $U_{p}$ and $U_{m}$ defined in (20) and (23), respectively, delimit reasonably well three different regimes of mixing. Although all the simulations use the electrovortex flow as a means to mix the alloy, we conjecture that the estimates $U_{p}$ and $U_{m}$ should be valid for generic fluid flows that could be generated by other mechanisms. More precisely, given some generic flow with the velocity scale $U$, we conclude the following:

(i) $U \ll U_{p}$ : The flow has no influence on the mixing of the alloy.

The flow is too weak to oppose buoyancy and therefore cannot affect the composition of the alloy at any time.

(ii) $U_{p} \ll U \ll U_{m}$ : The flow has a moderate influence on the mixing of the alloy. time.

The flow can oppose buoyancy and affects the composition of the alloy for a finite amount of

(iii) $U_{m} \ll U$ : The flow has a significant effect on the mixing of the alloy.

The flow overcomes buoyancy and affects the composition of the alloy at all times.

Of course the precise way the flow can affect the mixing depends on the flow itself. Nevertheless, we think that the above criteria based on (20) and (23) can be useful to make some first-order extrapolations to other cells. 


\section{Can nonswirling electrovortex flows mix alloys in LMBs?}

In this section we illustrate how the mixing criteria introduced above can be used. We have seen that for the cell under consideration in the paper and with the gravity $g=9.81 \mathrm{~m} \mathrm{~s}^{-2}$ the electrovortex mechanism cannot prevent the formation of the stratified buoyacy layer. But can the nonswirling electrovortex be used in other configurations to significantly affect the composition of the alloy during discharge? We have four parameters at our disposal: $H, R, R_{w}$, and $J$. For simplicity, we suppose in what follows that $H \approx R$ since the shallower the configuration the weaker the electrovortex flow. We also assume that the electrovortex is not swirling.

Let us first estimate $U$ in terms of the given parameters. In the inertial regime (or high-Reynoldsnumber regime, i.e., $S$ is large), we can use the following scaling law for the magnitude of the electrovortex flow in cylinders [29]:

$$
U \approx \sqrt{\frac{\mu_{0}}{\rho}} \frac{|J| R}{2} C, \quad \text { with } C=\left(10^{3-5 r_{w}}\right)^{1 / 3} .
$$

Here $r_{w}=R_{w} / R \leqslant 1$ is the relative radius of the wire with respect to the radius of the fluid domain. Notice that $C$ converges to its maximal value $C \rightarrow 10$ as $r_{w} \rightarrow 0$. Inserting this scaling law in the mixing criteria and using $H \approx R$, we find that the condition $U \ll U_{p}$ is equivalent to

$$
|J| R C^{3 / 2} \ll 4\left(\frac{\chi g \mathcal{M}_{\mathrm{Li}}}{n_{e} F}\right)^{1 / 2} \frac{\rho_{*}^{1 / 4}}{\mu_{0}^{3 / 4}} \Leftrightarrow \text { no influence on mixing, }
$$

and that the condition $U \gg U_{m}$ is equivalent to

$$
|J| R^{2 / 3} C^{5 / 3} \gg \frac{8}{\pi^{1 / 3}} \frac{\rho_{*}^{1 / 6}}{\mu_{0}^{5 / 6} D^{1 / 3}}\left(\frac{\chi \mathcal{M}_{\mathrm{Li}} g}{n_{e} F}\right)^{2 / 3} \Leftrightarrow \quad \text { significant effect on mixing. }
$$

The idea here is to write all the controllable parameters on the left of the inequalities, and all the physical constants that depend on the type of LMB that is used, on the right. Hence, for $\mathrm{Li} \| \mathrm{Pb}$ cells, we can say with good confidence that the electrovortex flow cannot affect the mixing if $|J| R C^{3 / 2} \ll$ $3.4 \mathrm{kA} \mathrm{m}^{-1}$, but it can efficiently mix the alloy if $|J| R^{2 / 3} \mathrm{C}^{5 / 3} \gg 500 \mathrm{kA} \mathrm{m}^{-4 / 3}$.

Recall that for the cell considered in the paper we have $r_{w}=0.2$ and $R=0.04 \mathrm{~m}$. The above argumentation leads us to conclude that in this cell there is no mixing if $J \ll J_{p}=8.6 \mathrm{kA} \mathrm{m}^{-2}$ and there is efficient mixing if $J \gg J_{m}=330 \mathrm{kA} \mathrm{m}^{-2}$. Both $J_{p}$ and $J_{m}$ are above the current densities we have used in the numerical simulations, and certainly $J_{m}$ is unachievable in realistic setups.

Let us now inspect the most optimistic scenario with $r_{w} \rightarrow 0$ to have the most intense electrovortex flow, and let us use the realistic value $J=-10 \mathrm{kA} \mathrm{m}^{-2}$. From the previous inequalities, we estimate that the electrovortex flow has no mixing effect in cells with radii smaller than $R_{p}=0.01 \mathrm{~cm}$ which is very small. However, to significantly affect mixing, the radius (and height) of the cell must be larger than $R_{m}=1.1 \mathrm{~m}$. Considering that this is the most optimistic scenario and that present day LMBs are far from reaching $1 \mathrm{~m}$ in size, we conclude that nonswirling electrovortex flows have very limited effect on the mixing of the alloy in small and large LMBs.

This conclusion only applies to nonswirling electrovortex flows. For swirling electrovortex flows, i.e., the electrovortex flows modified by an ambient magnetic field, the simulations reported in Ref. [9] suggest that efficient mixing of the alloy can be achieved even in small cells. We have observed the same phenomenon in preliminary numerical simulations and but this question remains a matter for future investigations.

\section{CHARGING CELL}

\section{A. Simulations}

We now focus our attention on the charging phase of the LMB. This is done in the numerical simulations by enforcing $J$ to be positive and by preparing appropriately the initial state of the 
electrode. We set the initial molar fraction to be $x_{\mathrm{Li}}=50 \%$, which corresponds a homogeneous alloy rich in lithium. Over time a constant flux of light $\mathrm{Li}$ is extracted from the alloy at the top of the electrode. This locally decreases the molar fraction $x_{\mathrm{Li}}$ and thus makes the alloy locally heavier than the bulk. As time increases, this inevitably creates an unstable stratification and triggers solutal convection: the heavy fluid parcels created at the interface with the electrode start to sink toward the bulk of the alloy. Solutal convection in charging Li||Bi cells was studied in Ref. [14] using axisymmetric simulations. Here we present new fully three-dimensional simulations in a slightly bigger and different $\mathrm{Li}|| \mathrm{Pb} \mathrm{LMB}$ than [14]. We keep the thin copper wire connected to the bottom of the cell; therefore, the electrovortex mechanism is active, but we will see that it is very weak.

We use the same nondimensional values of $S$ as in Sec. III A, that is $S \in\left\{10,10^{3}, 10^{5}\right\}$. Since the reference density $\rho_{*}$ is smaller than in the discharge scenario [see (3b)], these choices for $S$ correspond to charging the battery with the current densities $J \in\{43,430,4300\} \mathrm{A} \mathrm{m}^{-2}$. Recall that since the simulations are three dimensional, the velocity field is approximated by using the composite expansion (11) with finite elements in the meridian section and Fourier modes in azimuth. In order to make diagnostics on the root-mean-square (rms) velocity we isolate the axisymmetric flow component $U_{\text {axi }}$ (Fourier mode $m=0$ ) and combine all the other components into $U_{3 \mathrm{D}}$ (Fourier modes $m \geqslant 1$ ):

$$
U_{\mathrm{axi}}=\sqrt{2 \pi \int_{0}^{R} \int_{0}^{H}\left\|\boldsymbol{u}_{0}^{c}\right\|^{2} r d r d z}, \quad U_{3 \mathrm{D}}=\sqrt{\pi \sum_{m=1}^{M-1} \int_{0}^{R} \int_{0}^{H}\left(\left\|\boldsymbol{u}_{m}^{c}\right\|^{2}+\left\|\boldsymbol{u}_{m}^{s}\right\|^{2}\right) r d r d z} .
$$

We show in Fig. 11 the time evolution of $U_{\text {axi }}$ and $U_{3 \mathrm{D}}$ with the low current density $J=43 \mathrm{~A} \mathrm{~m}^{-2}$. To better interpret these graphs, we also show in this Fig. 5 snapshots [marked (i) to (v)] of the molar fraction and of the velocity field (magnitude and streamlines). After a short transient phase, we observe in panel (a) that $U_{\text {axi }}$ reaches a first plateau with $U_{\text {rms }} \sim 0.2 \mu \mathrm{m} \mathrm{s}^{-1}$. The state corresponding to this plateau is shown in the snapshot (i). The flow is dominated by the electrovortex mechanism, but the electrovortex flow is weak and does not significantly affect the molar fraction. We nevertheless refer to this state later as the electrovortex plateau. At $t_{\text {conv }} \approx 33 \mathrm{~s}$ [star in Fig. 11(a)] a transition to a more intense convective flow state occurs. The snapshot (ii) shows the state of the flow near the end of this transition. We see that small-scale structures emerge near the upper interface and fall down with typical plumelike shape. After some time, these plumes reorganize and the flow settles into a state characterized by a strong vertical jet flowing downward along the axis as seen in the snapshot (iii). Up to this point the dynamics is still axisymmetric. After that the convective flow gradually evolves toward three-dimensionality but keeps the same magnitude. We denote $U_{\text {axi }}^{\infty}$ the amplitude of the axisymmetric component of the flow in this regime. The snapshots (iv) and (v) show the flow right before and right after the transition to three dimensionality. Notice the typical cellular structure of the convection cells in the snapshot (v). Since these fully three-dimensional simulations are computationally demanding, we did not run them for very long. In the present simulation the three-dimensional structures grow from the numerical noise and therefore take a long time to emerge. Since in actual experiments, the background noise level is significantly larger than in the numerical simulations, it is likely that three-dimensional convection occurs shortly after $t_{\text {conv }}$ in reality. In Fig. 12(a), we show results from axisymmetric simulations with $J \in\{43,430,4300\} \mathrm{A} \mathrm{m}^{-2}$. We observe that the higher $J$ the higher the initial electrovortex flow plateau. We also observe that the transition to solutal convection occurs earlier and that a more intense convective flow is present. We can estimate from these three curves typical values for $t_{\text {conv }}$ and $U_{\text {axi }}^{\infty}$. These estimations are reported as functions of $J$ in Figs. 12(b) and 12(c). Although there are only three datapoints, two decades are covered and we clearly observe the power-law behavior

$$
t_{\mathrm{conv}} \sim J^{-2 / 5}, \quad U_{\mathrm{axi}}^{\infty} \sim J^{2 / 5}
$$



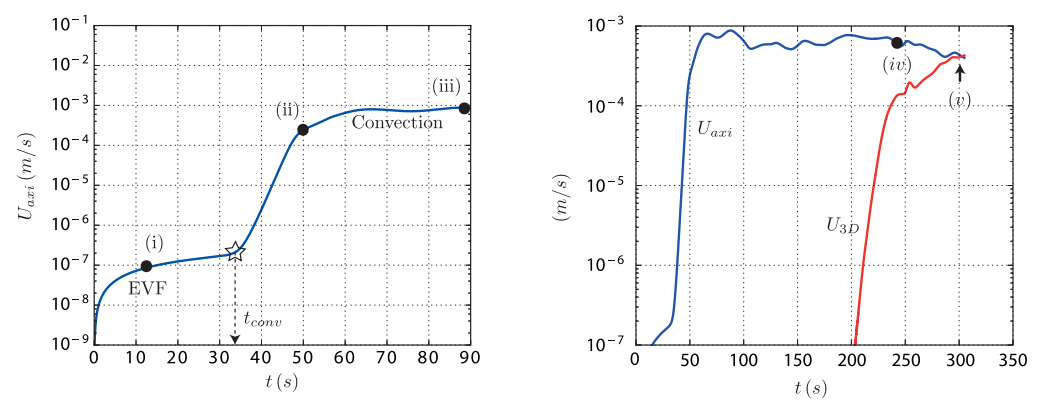

(a) rms velocity

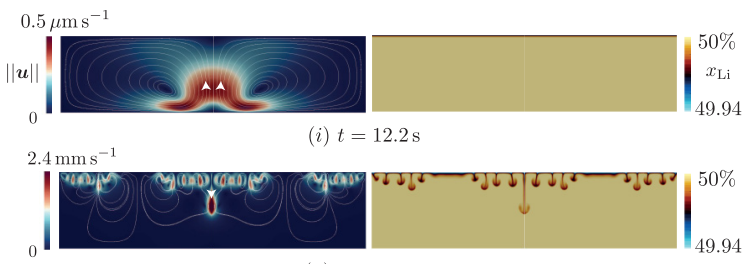

(ii) $t=50.0 \mathrm{~s}$

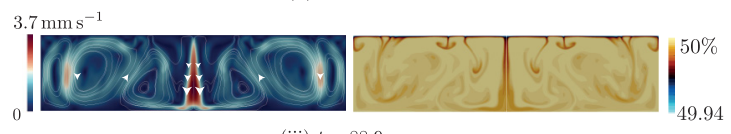

(iii) $t=88.9 \mathrm{~s}$

(b) snapshots of flow intensity and molar fraction

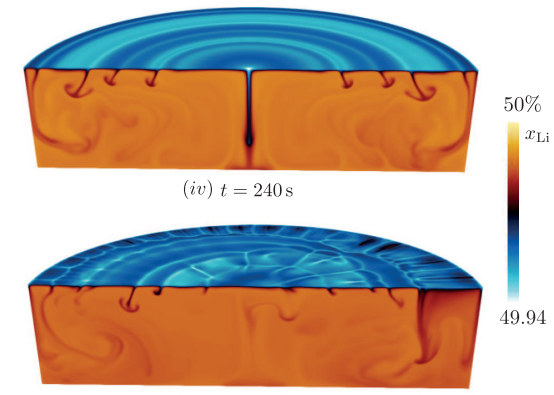

(v) $t=300 \mathrm{~s}$

FIG. 11. Charging the cell with the weak current density $J=43 \mathrm{Am}^{-2}$. (a) Time evolution of rms velocities $U_{\text {axi }}$ and $U_{3 \mathrm{D}}$. (b) Snapshots of the molar fraction and of the velocity streamlines and magnitude at different times. The electrovortex flow is visible at very short times, but after $t_{\text {conv }}$ solutal convection takes over the dynamics and typical plumelike structures fall downward. The flow is first mainly two-dimensional and dominated by a central downward jet, but at later times the convective flow becomes three-dimensional and develops a typical cellular structure.

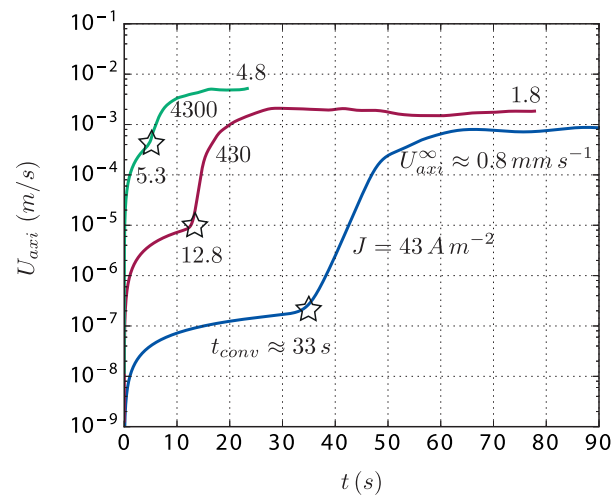

(a)

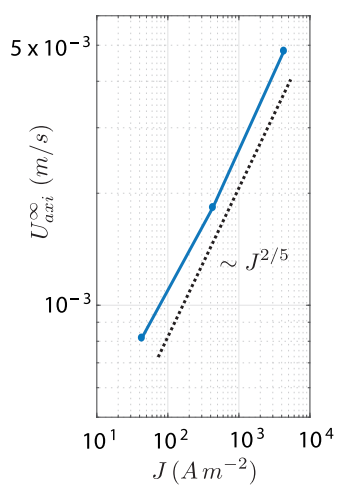

(b)

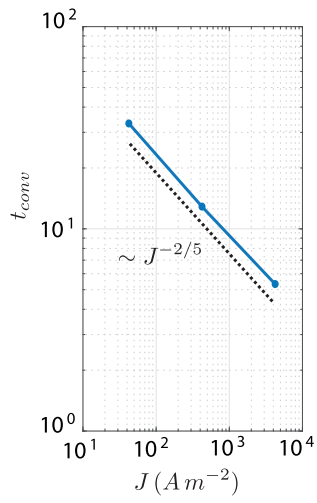

(c)

FIG. 12. Axisymmetric simulations of the charging cell with $J \in\{43,430,4300\} \mathrm{A} \mathrm{m}^{-2}$. (a) Time evolution of the rms velocity. From these curves we estimate the critical time for the onset of solutal convection (marked by star), $t_{\text {conv }}$, and the late time rms speed $U_{\text {axi }}^{\infty}$ of the flow. (b) $U_{\text {axi }}^{\infty}$ as a function of $J$ (c) $t_{\text {conv }}$ as a function of $J$. Observe the power law-behaviors $U_{\mathrm{axi}}^{\infty} \sim J^{2 / 5}$ and $t_{\mathrm{conv}} \sim J^{-2 / 5}$. 


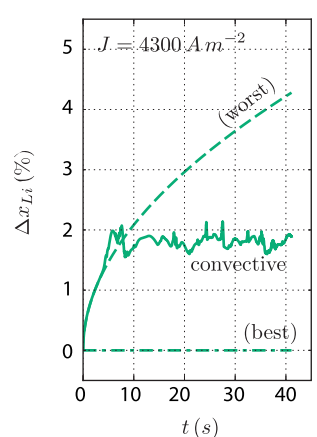

(a) inhomogeneity

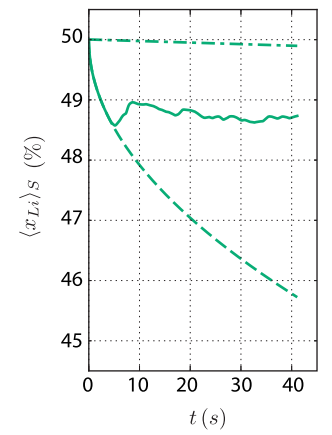

(b) surface molar fraction

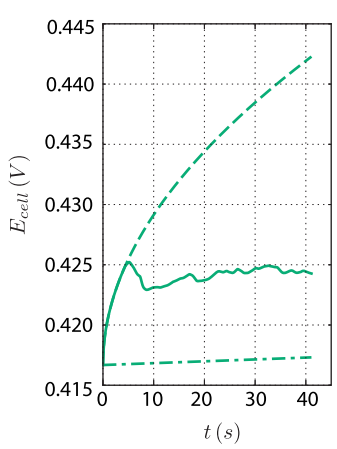

(c) cell voltage

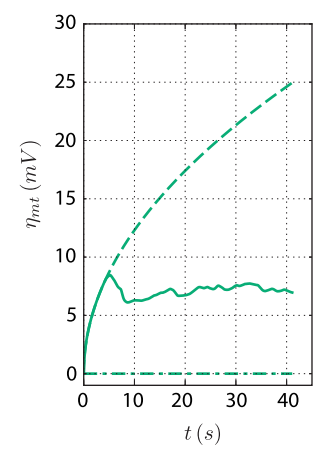

(d) overpotential

FIG. 13. Charging cell with fixed current density $J=4300 \mathrm{~A} \mathrm{~m}^{-2}$. We quantitively compare the numerical simulations (full lines) to the worst-case scenario (pure diffusion, dashed lines) and to the best-case scenario (perfect blend, dot-dashed lines). (a) Inhomogenity in the cell measured by $\Delta x_{\mathrm{Li}}=\max \left(x_{\mathrm{Li}}\right)-\min \left(x_{\mathrm{Li}}\right)$. (b) Average composition on the interface $\left\langle x_{\mathrm{Li}}\right\rangle_{s}$. (c) Theoretical cell voltage $E_{\text {cell }}$. (d) Mass transfer overpotential $\eta_{\mathrm{mt}}$.

Notice that the 2/5 exponent in the velocity scale is similar to the one we have derived in the estimate of $U_{m}$ [see (23)]. This is not a surprise since a similar argument based on energy balance can be invoked to explain these scaling laws, as we show below.

In Fig. 13, we provide further quantitive information regarding mixing efficiency using $J=$ $4300 \mathrm{~A} \mathrm{~m}^{-2}$. We adopt the same presentation as in Fig. 4. Next to the numerical simulations (full lines), we plot the results (dashed) corresponding to the worst-case scenario (purely diffusive solution) and the results (dot-dashed) from the best-case scenario (perfectly blended homogeneous alloy). All the profiles initially follow the diffusive solution up until the time $t_{\text {conv }} \approx 5.3 \mathrm{~s}$ which corresponds to the onset of solutal convection. After that, the alloy is very efficiently blended by the flow as we observe in Fig. 13(a): The inhomogeneity in the molar fraction remains roughly constant and is about $\Delta x \approx 2 \%$ thereafter. This efficient mixing has a strong impact on the average molar fraction at the interface with the electrolyte [Fig. 13(b)]: The surface average at the interface is only $1.5 \%$ away from the volume-averaged molar fraction. As a result, the potential of the cell remains very close to what one would have in the best-case scenario [Fig. 13(c)] and so the mass transfer overpotential is always very low [Fig. 13(d)].

\section{B. Estimates for typical flow speed, mixing time, and typical inhomogeneity in the alloy}

The present simulations of a charging battery suggest that solutal convection requires a certain amount of time to be active, $t_{\text {conv }}$, and after this time, convection drives a certain flow intensity, $U$, which we now estimate.

In a first unsuccessful attempt, we tried to estimate $t_{\text {conv }}$ on the basis of a linear stability argument. The reasoning goes as follows. At very short times, the diffusive solution holds [see (16b)] even for an unstable stratification. Using this profile, we estimate the typical height of the stratified layer to be $h(t) \approx 2 \sqrt{D t}$ and the density difference between the top interface $z=H$ and the bulk of the alloy to be of the order

$$
\Delta \rho(t) \approx 2 \chi s_{\mathrm{Li}} \sqrt{\frac{t}{\pi D}} .
$$

With this, we introduce a local Rayleigh number

$$
\operatorname{Ra}(t)=\frac{g \Delta \rho h^{3}}{\rho_{*} v D}=\frac{16}{\sqrt{\pi}} \frac{s_{\mathrm{Li}} \beta g t^{2}}{v}=\frac{16}{\sqrt{\pi}} \frac{J \mathcal{M}_{\mathrm{Li}} \beta g t^{2}}{n_{e} F v},
$$


which apparently grows quadratically in time. At some critical time $t_{c}$, a critical value for the onset of solutal convection is reached $\mathrm{Ra}_{c}=\mathrm{Ra}\left(t_{c}\right)$ (typically in Rayleigh-Bénard convection, $\left.\mathrm{Ra}_{c} \in\left[10^{3}, 5 \times 10^{3}\right][30]\right)$ and after that, one expects the unstably stratified layer to be quickly destabilized. This argument unavoidably yields the power law $t_{c} \sim J^{-1 / 2}$ which is not observed. Linear stability also does not allow to estimate the intensity of the convective flow and is therefore not adapted to explain our observations.

Our second idea, which turned out to be more successful, is closely related to the argument we developed for the derivation of $U_{m}$. Solutal convective motions occur because available gravitational potential energy is being transformed into kinetic energy. With $\Delta \rho(t)$ as in (28), we conjecture that the following balance:

$$
\frac{\rho_{*} U(t)^{2}}{2} \approx \Delta \rho(t) g H
$$

is useful to define the velocity scale $U(t)$. The difference with respect to the discharge scenario is that it is the density difference that actually drives the flow. Since the heavy plumes created at the interface with the electrolyte are accelerated by gravity until they reach the bulk of the alloy, we use $H$ as length scale on the right-hand side of (30). As Fig. 13(a) suggests, the inhomogeneity level, and so also $\Delta \rho$, saturates after a typical advection time $t=H / U$ since this is indeed the time that is needed to blend the plumes into the bulk once they have left the top interface. Recalling (28) and using $\Delta \rho(H / U)$ in (30), the energy density balance yields

$$
U=C_{u}\left(\frac{\chi J \mathcal{M}_{\mathrm{Li}} g}{n_{e} F \rho_{*}}\right)^{2 / 5} \frac{H^{3 / 5}}{D^{1 / 5}}
$$

and, from $T=H / U$, we find

$$
T=C_{t}\left(\frac{n_{e} F \rho_{*} H}{\chi J \mathcal{M}_{\mathrm{Li}} g}\right)^{2 / 5} D^{1 / 5} .
$$

Comparing (31a) and (23), we conclude that the solutal convection flow settles at the velocity scale similar to the quantity $U_{m}$ we have encountered in the discussion on the discharge scenario. Here we have introduced two geometry dependent constants $C_{u}, C_{t}$, which we can estimate from the numerical simulations. Using the material parameters of the fluid and $\rho_{*}=\rho_{50 \%}$, we find $C_{u}=0.145 \pm 0.008$ by setting $U=U_{\text {axi }}^{\infty}$, and we find $C_{t}=8.7 \pm 0.2$ by setting $T=t_{\text {conv }}$. More simulations varying the other parameters are required to ascertain these relations. We further estimate the width of the stratified top layer as

$$
\delta=\sqrt{D T}=C_{t}^{1 / 2}\left(\frac{n_{e} F \rho_{*} H}{\chi J \mathcal{M}_{\mathrm{Li}} g}\right)^{1 / 5} D^{3 / 5} .
$$

The remaining inhomogeneity level in the alloy is estimated to be that of the purely diffusive solution at time $t \approx T$ :

$$
\Delta \rho_{\mathrm{Li}} \approx 2 \sqrt{\frac{C_{t}}{\pi}}\left(\frac{\chi J \mathcal{M}_{\mathrm{Li}}}{n_{e} F}\right)^{4 / 5}\left(\frac{\rho_{*} H}{g}\right)^{1 / 5} D^{-2 / 5} .
$$

Notice that in the above physics-based argument the viscosity of the alloy appears to have no influence on the estimates $U, T, \delta$, and $\Delta \rho_{\mathrm{Li}}$. We conjecture that this could be a consequence of the Schmidt number $\mathrm{Sc}=v / D=18 \gg 1$ being large. Notice finally that the same scaling laws can be found from the three term balance:

$$
\left[\rho \partial_{t} \boldsymbol{u}\right] \sim[\rho(\boldsymbol{u} \cdot \nabla) \boldsymbol{u}] \sim \Delta \rho g \quad \Leftrightarrow \quad \frac{\rho_{*} U}{T} \sim \frac{\rho_{*} U^{2}}{H} \sim \frac{2 \chi|J| \mathcal{M}_{\mathrm{Li}} g}{n_{e} F} \sqrt{\frac{T}{\pi D}}
$$


coming from the momentum equation. In a low viscosity fluid, this type of equilibrium seems justified for thin plumelike structures that form at the top of electrode and accelerate downward until they reach the bottom of the cell.

\section{CONCLUSION}

In this article we have studied the composition and the motion of the alloy composing the positive electrode of a cylindrical LMB. We have studied the influence of solutal buoyancy and of the electrovortex mechanism in the discharge phase and in the charge phase. We have combined state-of-the-art numerical simulations with simple theoretical arguments, and derived scaling laws that provide insights in the physics that governs the flow and the mixing process.

In small cells we have observed that during discharge the electrovortex flow is active in the bulk, but this flow hardly penetrates the stagnant stratified layer of lighter fluid that slowly grows at the interface with the electrolyte. Due to the stabilizing effect of solutal buoyancy, the only active process homogenizing $\mathrm{Li}$ is molecular diffusion. This was also observed in Ref. [14]. Using simple energy balance arguments, we have derived formulas for two characteristic flow intensities $U_{p}$ and $U_{m}$ that define three regimes in the mixing process. Any flow with a velocity scale $U$ such that $U \ll U_{p}$ cannot counter solutal buoyancy and therefore cannot prevent the formation of a buoyant stagnant layer at the interface with the electrolyte. If $U \gg U_{m}$, then the flow can significantly affect the composition of the alloy and might mix the alloy efficiently. If $U_{p} \ll U \ll U_{m}$, then the flow moderately influences the mixing. These theoretical estimates are not restricted to fluid flows generated by the electrovortex mechanism and can be useful in other contexts of flow-induced mixing. We have tested the relevance of these estimates by running simulations with various gravity coefficients. By combining the formulas for $U_{p}$ and $U_{m}$ with previously known scaling laws for the intensity of the electrovortex flow, assuming that the electrovortex flow does not swirl [29], we conclude that nonswirling electrovortex flows cannot significantly mix the alloy in cells of realistic size. The situation is different for swirling electrovortex flows since in this case, according to Ref. [9], efficient mixing of the alloy can occur in the positive electrode. We intend to investigate this configuration in the near future.

During charge, we have observed that the electrovortex flow is active only for a short time $t_{\text {conv }}$, after which intense solutal convection takes over. As a result of the strong solutal convection the alloy is efficiently mixed. This observation has also been reported in Ref. [14] in axisymmetric simulations of solutal convection in $\mathrm{Li}|| \mathrm{Bi}$ cells. We have also observed that once solutal convection takes place the flow eventually becomes fully three-dimensional even for low charging currents. By varying the electrical current passing through the cell, we have observed the following scaling laws for the typical flow speed $\left(U \sim J^{2 / 5}\right)$ and time required for the onset of compositional convection (or mixing time) $\left(t_{\text {conv }} \sim J^{-2 / 5}\right)$. These scaling laws cannot be explained from linear stability arguments. They can be derived from simple energy balance arguments similar to those that led us to define the characteristic flow velocity $U_{m}$. These scaling laws can be used to estimate the mixing time and the inhomogeneity level in the alloy in other regimes of operation and in other LMBs.

An obvious extension to this work is a more detailed study of the action of the swirling electrovortex flows proposed in Ref. [9]. We expect that energy balance arguments similar to those we have developed here should allow us to predict the mixing capabilities of these flows, and we intend to communicate on this in a future work. Another possible extension consists of improving our LMB model by incorporating electrochemical restrictions. In the present work, but also in Refs. [9,12-14], it is assumed that a constant current density enters the cell at the top of the alloy. But at the interface with the salt, the local current density may well depend on the local composition of the alloy (see the model of Ref. [18]) and this effect is entirely ignored here. More investigation on this subject seems mandatory. 


\section{ACKNOWLEDGMENTS}

This work was supported by the Deutsche Forschungsgemeinschaft (DFG, German Research Foundation) by Award No. 338560565. Fruitful discussions with A. Sergent (LIMSI), T. Weier, and N. Weber (HZDR) are gratefully acknowledged. The HPC resources were provided by GENCIIDRIS (Grant No. 2019-0254) in France and by the Texas A\&M University Brazos HPC cluster. J.-L.G. acknowledges support from University Paris Saclay, the National Science Foundation, under Grants No. NSF DMS 1620058, No. DMS 1619892, the Air Force Office of Scientic Research, USAF, under Grant/Contract No. FA9550-18-1-0397, and the Army Research Office under Grant/Contract No. W911NF-15-1-0517.

\section{APPENDIX A: LI-PB ALLOYS: MATERIAL PROPERTIES AND FUNDAMENTAL EVOLUTION LAWS}

The $\mathrm{Li} \| \mathrm{Pb}$ cell considered in this article was studied in the 1960s at the Argonne National Laboratory and it is the basis for the more recent and more efficient $\mathrm{Li} \| \mathrm{Pb}-\mathrm{Sb}$ batteries $[1,2,31]$. $\mathrm{Li} \| \mathrm{Pb}$ cells are unlikely to be commercialized, but they are very well adapted to fundamental studies, mainly because material properties of $\mathrm{Li}-\mathrm{Pb}$ alloys are well known (these alloys are planned to be used in tritium breeders of nuclear fusion reactors, see Refs. [16,32-35]). We discuss here the main material properties of the alloy and explain the hypotheses behind the model used in the present paper.

To keep track of the composition of the alloy, one can use many different variables:

$$
\begin{aligned}
& \text { molar concentrations: } \quad c_{\mathrm{Li}}, c_{\mathrm{Pb}}, \quad c=c_{\mathrm{Li}}+c_{\mathrm{Pb}} \quad\left(\text { in } \mathrm{mol} \mathrm{m}^{-3}\right) \text {, } \\
& \text { mass concentrations: } \rho_{\mathrm{Li}}, \rho_{\mathrm{Pb}}, \quad \rho=\rho_{\mathrm{Li}}+\rho_{\mathrm{Pb}} \quad\left(\text { in } \mathrm{kg} \mathrm{m}^{-3}\right) \text {, } \\
& \text { molar fractions: } x_{\mathrm{Li}}=c_{\mathrm{Li}} / c, \quad x_{\mathrm{Pb}}=1-x_{\mathrm{Li}} \quad(\text { in } \%) \text {, } \\
& \text { mass fractions: } \omega_{\mathrm{Li}}=\rho_{\mathrm{Li}} / \rho, \quad \omega_{\mathrm{Pb}}=1-\omega_{\mathrm{Li}} \quad \text { (in \%). }
\end{aligned}
$$

Molar and mass concentrations are related as follows: $\rho_{\mathrm{Li}}=\mathcal{M}_{\mathrm{Li}} c_{\mathrm{Li}}$ and $\rho_{\mathrm{Pb}}=\mathcal{M}_{\mathrm{Pb}} c_{\mathrm{Pb}}$ (unit $\mathrm{kg} / \mathrm{m}^{3}$ ), where $\mathcal{M}_{\mathrm{Li}}$ and $\mathcal{M}_{\mathrm{Pb}}$ are the molar masses of $\mathrm{Li}$ and $\mathrm{Pb}$. Of all these variables, only two are independent. In our study we have used $\rho_{\mathrm{Li}}$, the mass concentration of $\mathrm{Li}$ in the alloy, and $\rho$, the total density. The relations (2) connecting $\rho_{\mathrm{Li}}$ and $\rho$ to the molar fraction $x_{\mathrm{Li}}$ are directly derived from the definitions above. In this study, we suppose that the Li-Pb alloy is at $500{ }^{\circ} \mathrm{C}$. According to the state diagram in Ref. [16] the alloy remains liquid in a wide interval of molar fraction $x_{\mathrm{Li}} \in[0,60] \%$. Beyond $x_{\mathrm{Li}}>60 \%$ solid intermetallic phases form. References $[16,17]$ provide experimental measures for density $\rho$ as a function of the molar fraction $x_{\mathrm{Li}}$ [inset of Fig. 14(a)]. Combined with (2), we can represent $\rho$ as a function of $\rho_{\mathrm{Li}}$, which is done in Fig. 14(a). This clearly suggests that a linear fit is adequate and leads to the fit adopted in (3a). The values of the kinematic viscosity, electrical conductivity, and binary diffusion coefficient we give in (6) are those of the eutectic mixture (see Refs. [34,35]). From Ref. [34], we infer that $D$ ranges nonuniformly in the interval $D \in[7.3,26.6] \times 10^{-9} \mathrm{~m}^{2} \mathrm{~s}^{-1}$ when the molar fraction is in the range $x_{\mathrm{Li}} \in[30,60] \%$ and the temperatures in the range $T \in[724,994] \mathrm{K}$. The variation of $\sigma$ and $v$ with changing composition is not documented. For simplicity, we use in the paper the eutectic alloy values over the entire range $x_{\mathrm{Li}} \in[17,60] \%$. This is obviously an approximation, but considering that $v$ and $D$ are always very small and $\sigma$ very large, we believe that this approximation is able to capture the relevant physical features of the problem. In Fig. 14(b) we plot the experimental data from Ref. [26] for the equilibrium potential $\Phi$ as a function of $x_{\mathrm{Li}}$. This plot suggests that the linear fit for $\Phi\left(x_{\mathrm{Li}}\right)$ given in (13) is adequate for the entire range $x_{\mathrm{Li}} \in[17,60] \%$ covered by our study. 


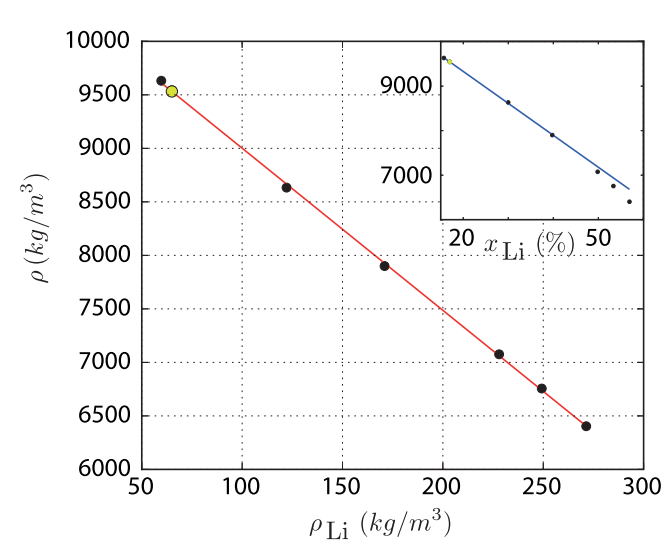

(a) density

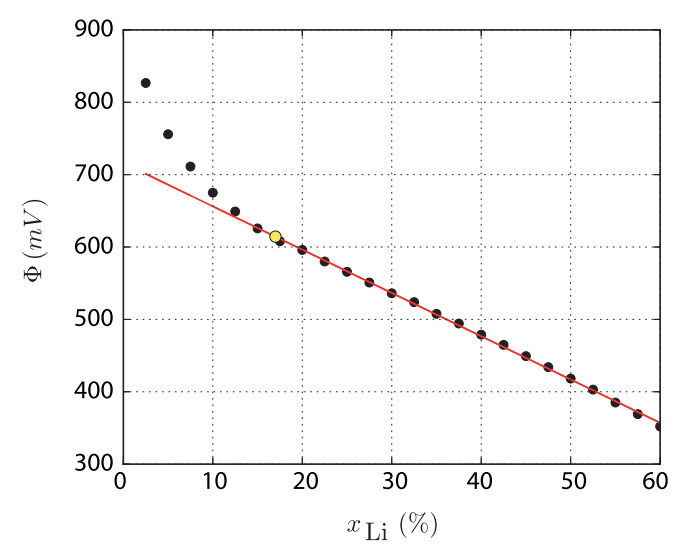

(b) thermodynamical potential

FIG. 14. Material properties of the Li-Pb alloys for varying composition. The clear dot highlights the eutectic mixture with $x_{\mathrm{Li}}=17 \%$. (a) The density of the alloy $\rho$ as a function of the mass concentration of lithium $\rho_{\mathrm{Li}}$ follows an approximatively linear law [see (3a) for fit]. (Inset: $\rho$ as a function of $x_{\mathrm{Li}}$ is less linear). (b) The thermodynamical equilibrium potential $\Phi$ follows a linear law [see (13) for fit] in the large interval $x_{\mathrm{Li}} \in[17,60] \%$.

We now turn our attention to the evolution equations for the composition of the alloy. On a fundamental level, we can express local conservation of species in the binary alloy through

$$
\begin{aligned}
\partial_{t} \rho_{\mathrm{Li}}+\nabla \cdot\left(\rho_{\mathrm{Li}} \boldsymbol{u}\right) & =\nabla \cdot\left[D \rho \nabla\left(\frac{\rho_{\mathrm{Li}}}{\rho}\right)\right], \\
\partial_{t} \rho_{\mathrm{Pb}}+\nabla \cdot\left(\rho_{\mathrm{Pb}} \boldsymbol{u}\right) & =\nabla \cdot\left[D \rho \nabla\left(\frac{\rho_{\mathrm{Pb}}}{\rho}\right)\right],
\end{aligned}
$$

where we accept that a diffusive process is active, see Ref. [36]. These evolution equations are expressed in terms of the mass concentrations $\rho_{\mathrm{Li}}$ and $\rho_{\mathrm{Pb}}$ so that $\rho=\rho_{\mathrm{Li}}+\rho_{\mathrm{Pb}}$ is the total mass density. It is important to notice that the diffusive term is expressed in terms of the mass fractions, $\rho_{\mathrm{Li}} / \rho, \rho_{\mathrm{Pb}} / \rho$ which is necessary to conserve the mass balance. Indeed, by summing both evolution laws and using $\rho_{\mathrm{Pb}} / \rho=1-\left(\rho_{\mathrm{Li}} / \rho\right)$, we find that

$$
\partial_{t} \rho+\nabla \cdot(\rho \boldsymbol{u}) \equiv 0 .
$$

Of the three previous equations, only two are independent. In the body of the text, we choose to keep $\rho_{\mathrm{Li}}$ and $\rho$ as independent variables and (A2a) and (A3) as evolution laws. In order to reach the equations presented in the article, we make further simplifying assumptions.

A first simplification can be done on the diffusive flux. Using (3a) i.e., $\nabla \rho \approx-\chi \nabla \rho_{L i}$, we have

$$
D \rho \nabla\left(\frac{\rho_{\mathrm{Li}}}{\rho}\right) \approx\left(1+\frac{\chi \rho_{\mathrm{Li}}}{\rho}\right) D \nabla \rho_{\mathrm{Li}} .
$$

We can further approximate this term to $\nabla \cdot\left(D \nabla \rho_{\mathrm{Li}}\right)$, but this obviously requires that

$$
\Pi_{1}=\frac{\chi \rho_{\mathrm{Li}}}{\rho} \ll 1
$$

For the Li-Pb alloys with $x_{\mathrm{Li}} \in[17,60] \%$, we find $\Pi_{1} \in[0.10,0.72]$, which implies that the approximation

$$
\partial_{t} \rho_{\mathrm{Li}}+\nabla \cdot\left(\rho_{\mathrm{Li}} \boldsymbol{u}\right) \approx \nabla \cdot\left(D \nabla \rho_{\mathrm{Li}}\right)
$$


used in all our simulations and in the previous investigations [9,14], is only accurate for alloys with low Li concentration. From (A4) it seems plausible to think that Li-rich regions have effective diffusion coefficients $\left(1+\Pi_{1}\right) D$ that are slightly larger than $D$, but this is without considering that $D$ may still vary with the composition of the alloy [34]. Assuming that $D$ is constant is likely to be a bigger assumption than assuming that the diffusive term is $\nabla \cdot\left(D \nabla \rho_{\mathrm{Li}}\right)$. In future work we plan to adopt the model (A2a) but we do not expect to see big differences since diffusion is always weak compared to the other mechanisms at play in the problem.

A second simplification consists of injecting the linear fit (3a) into the total mass balance (A3). Then, using (A6), we find that

$$
\boldsymbol{\nabla} \cdot \boldsymbol{u} \approx \underbrace{\frac{\chi \nabla \cdot\left(D \nabla \rho_{\mathrm{Li}}\right)}{\rho_{*}+\chi \rho_{\mathrm{Li}, *}}}_{\text {small }} .
$$

Considering the small value of the diffusion coefficient $D$ and the large value of $\rho_{0 \%}=\rho_{*}+$ $\chi \rho_{\mathrm{Li}, *}=10525 \mathrm{~kg} \mathrm{~m}^{-3}$, which is nearly the density of pure $\mathrm{Pb}$, the right-hand side seems very small. This suggests that the flow is almost incompressible. More precisely, we need to have

$$
\left[\frac{\chi \nabla \cdot\left(D \nabla \rho_{\mathrm{Li}}\right)}{\rho_{*}+\chi \rho_{\mathrm{Li}, *}}\right] \ll\left[\frac{1}{r} \frac{\partial\left(r u_{r}\right)}{\partial r}\right],\left[\frac{\partial u_{z}}{\partial z}\right] \Leftrightarrow \Pi_{2}=\frac{\chi D \rho_{\mathrm{Li}}}{\rho_{0 \%} U R} \ll 1
$$

in cylindrical coordinates. Using $U=1 \mathrm{~cm} \mathrm{~s}^{-1}$ as velocity reference scale, $R=4 \mathrm{~cm}$ as length scale, and $\rho_{\mathrm{Li}} \in[65,292] \mathrm{kg} \mathrm{m}^{-3}$, we find $\Pi_{2} \in[1.9,8.4] \times 10^{-6}$ which is indeed very small. In conclusion, the assumption of incompressibility is always very well justified in the binary alloy:

$$
\nabla \cdot \boldsymbol{u} \approx 0 .
$$

This means in particular that we can further write $\nabla \cdot\left(\rho_{\mathrm{Li}} \boldsymbol{u}\right) \approx \boldsymbol{u} \cdot \nabla \rho_{\mathrm{Li}}$ in the evolution equation for $\rho_{\mathrm{Li}}$.

The Boussinesq hypothesis is the last simplifying assumption we need to investigate. Recalling that the inertial term in the momentum equation is $\rho\left(\partial_{t} \boldsymbol{u}+\boldsymbol{u} \cdot \nabla \boldsymbol{u}\right)$, we can simplify $\rho$ to $\rho_{*}$ only if

$$
\Pi_{3}=\frac{\left(\rho-\rho_{*}\right)}{\rho_{*}} \ll 1 .
$$

During charge, solutal convection prevents large differences in density so that a Boussinesq model with fixed $\rho_{*}$ is always well adapted to simulate short time behavior. During discharge, strong density differences can build up according to our simulations. Potentially these density differences can be as high as $\rho-\rho_{*} \approx 3000 \mathrm{~kg} \mathrm{~m}^{-3}$ according to Fig. 14(a), which is not so small compared to $\rho_{*}=9543 \mathrm{~kg} \mathrm{~m}^{-3}$. But the Boussinesq hypothesis nevertheless remains accurate because these large density differences were observed only in the numerical simulations of discharge and only in the almost stagnant buoyant layer where $\boldsymbol{u} \approx \mathbf{0}$. Hence, although $\Pi_{3}$ may not be so small, the whole inertial term is actually negligible in this case.

\section{APPENDIX B: HYDRODYNAMIC BOUNDARY CONDITIONS AT THE SALT-ALLOY INTERFACE}

All the numerical simulations presented in the paper are restricted to the alloy layer, and at boundary $z=H$, which is the interface with the electrolyte, we have imposed the no-slip conditions

$$
\text { no-slip : }\left.\boldsymbol{u}\right|_{z=H}=\mathbf{0} \text {. }
$$

An alternative choice could have been to use the free-slip conditions:

$$
\text { free-slip : }\left.u_{z}\right|_{z=H}=0,\left.\quad \partial_{z} \boldsymbol{u}_{\perp}\right|_{z=H}=0 .
$$

In fact, what we really have at $z=H$, is an interface between two liquids, the alloy and the salt. We study here the impact of using idealized free-slip or no-slip conditions and how this compares 


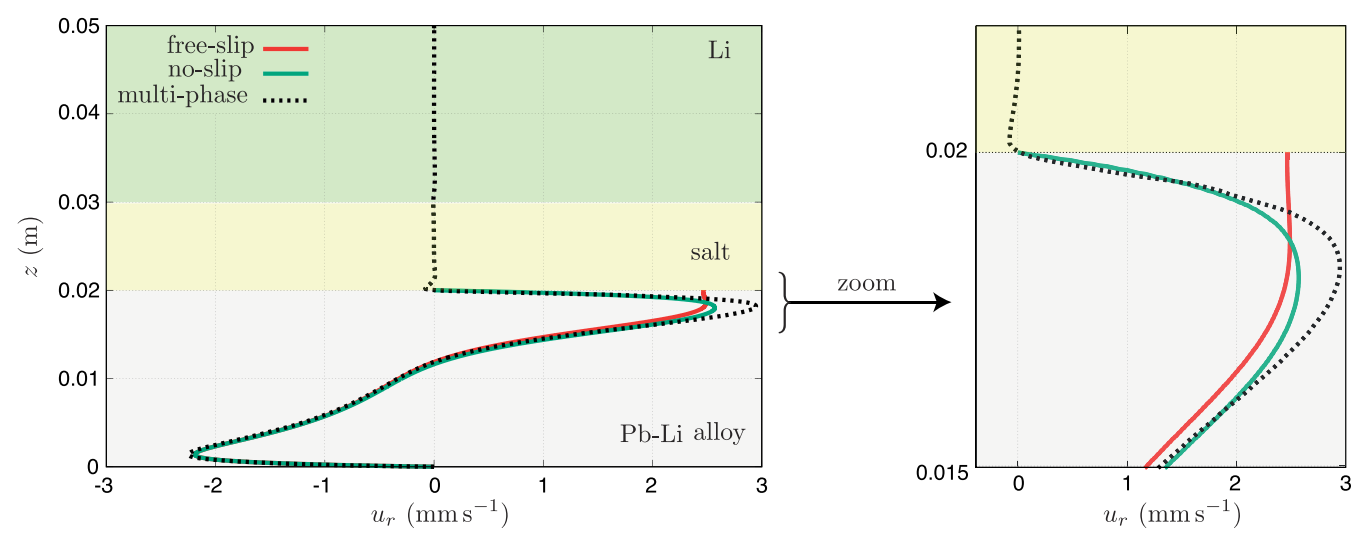

FIG. 15. Influence of hydrodynamical boundary conditions at the electrolyte-alloy interface. We compare numerical simulations using idealized boundary conditions at $z=H$, either free-slip or no-slip, to full multiphase simulations over the entire cell.

to the more realistic interfacial condition (continuity of the velocity and continuity of the normal component of stress tensor).

We perform three simulations of the battery in the discharging phase using $J=-5000 \mathrm{~A} \mathrm{~m}^{-2}$. In the first two simulations we operate as described in the paper. The domain is restricted to the positive electrode and we start with the eutectic alloy, i.e., $x_{\mathrm{Li}}=17 \%$. In the first simulation we enforce the no-slip boundary condition, and in the second one we enforce the free-slip boundary condition. We compare these single phase simulations to a more realistic multiphase simulation with three layers of fluid as was done in Ref. [15]. In this multiphase configuration, we place a salt layer above the alloy in the interval $z \in[2,3] \mathrm{cm}$, and a layer of pure lithium in the interval $z \in[3,5] \mathrm{cm}$. The material properties in the electrolyte and the negative electrode are as follows:

$$
\begin{aligned}
& \left(\rho_{\text {salt }}, \sigma_{\text {salt }}, v_{\text {salt }}\right)=\left(1621 \mathrm{~kg} \mathrm{~m}^{-3}, 187 \mathrm{~S} \mathrm{~m}^{-1}, 1.48 \times 10^{-6} \mathrm{~m}^{2} \mathrm{~s}^{-1}\right) \\
& \left(\rho_{\text {top }}, \sigma_{\text {top }}, v_{\text {top }}\right)=\left(486.4 \mathrm{~kg} \mathrm{~m}^{-3}, 2.94 \times 10^{6} \mathrm{~S} \mathrm{~m}^{-1}, 6.72 \times 10^{-7} \mathrm{~m}^{2} \mathrm{~s}^{-1}\right)
\end{aligned}
$$

The three layers are treated as a single fluid medium, that is, no boundary condition is enforced across the interfaces and the interfaces are allowed to move freely (the continuity of the velocity and of the normal component of the stress tensor are naturally enforced). We let the electrical current enter the cell uniformly at the top of the lithium layer. The current exits the positive electrode through a copper wire connected to the bottom of the electrode as described in the paper. All the other external boundaries are insulating as in Ref. [15] and we suppose axisymmetry. The initial state is rest for the three simulations. Since the vertical component of the velocity is always close to zero near the interface in the three models, we focus on the the velocity component $u_{r}$. We compare in Fig. 15 the values of $u_{r}$ obtained by the three methods at $r=8 \mathrm{~mm}$ and $t=10 \mathrm{~s}$. We observe that the velocity field is very weak in the negative electrode and in the electrolyte since the electrovortex flow is active only in the positive electrode (i.e., the alloy). The velocity profiles in the three cases are similar. We observe significant differences only in a thin boundary layer just below the interface between the electrolyte and the alloy. In this boundary layer, the radial flow in the multiphase simulation is slightly more intense than in the other two simulations. But we observe that the no-slip profile follows better the multiphase simulation than the free-slip profile. This suggests that the no-slip condition is a better approximation of the full multiphase setting than the free-slip condition when the simulations are restricted to a single phase and to the alloy layer. 
[1] E. J. Cairns, C. E. Crouthamel, A. K. Fischer, M. S. Foster, J. C. Hesson, C. E. Johnson, H. Shimotake, and A. D. Tevebaugh, Galvanic cells with fused-salt electrolytes, Report No. ANL-7316 (Argonne National Laboratory, US, 1967).

[2] H. L. Chum and R. A. Osteryoung, Review of thermally regenerative electrochemical cells (Solar Energy Research Institute, 1981), pp. 332-416.

[3] D. J. Bradwell, H. Kim, A. H. C. Sirk, and D. R. Sadoway, Magnesium-antimony liquid metal battery for stationary energy storage, J. Am. Chem. Soc. 134, 1895 (2012).

[4] H. Kim, D. A. Boysen, T. Ouchi, and D. R. Sadoway, Calcium-bismuth electrodes for large-scale energy storage (liquid metal batteries), J. Power Sources 241, 239 (2013).

[5] K. Wang, K. Jiang, B. Chung, T. Ouchi, P. J. Burke, D. A. Boysen, D. J. Bradwell, H. Kim, U. Muecke, and D. R. Sadoway, Lithium-antimony-lead liquid metal battery for grid-level energy storage, Nature 514, 348 (2014).

[6] D. H. Kelley and T. Weier, Fluid mechanics of liquid metal batteries, Appl. Mech. Rev. 70, 020801 (2018).

[7] N. Weber, V. Galindo, F. Stefani, and T. Weier, Current-driven flow instabilities in large-scale liquid metal batteries, and how to tame them, J. Power Sources 265, 166 (2014).

[8] X. Ning, S. Phadke, B. Chung, H. Yin, P. Burke, and D. R. Sadoway, Self-healing li-bi liquid metal battery for grid-scale energy storage, J. Power Sources 275, 370 (2015).

[9] W. Weber, M. Nimtz, P. Personnettaz, T. Weier, and D. Sadoway, Numerical simulation of mass transfer enhancement in liquid metal batteries by means of electro-vortex flow, J. Power Sources Adv. 1, 100004 (2020).

[10] D. H. Kelley and D. R. Sadoway, Mixing in a liquid metal electrode, Phys. Fluids 26, 057102 (2014).

[11] R. F. Ashour and D. H. Kelley, Convection-diffusion model of lithium-bismuth liquid metal batteries, in Proceedings of the TMS Annual Meeting \& Exhibition (Springer, Berlin, 2018), pp. 41-52.

[12] R. F. Ashour, D. H. Kelley, A. Salas, M. Starace, N. Weber, and T. Weier, Competing forces in liquid metal electrodes and batteries, J. Power Sources 378, 301 (2018).

[13] N. Weber, M. Nimtz, P. Personnettaz, A. Salas, and T. Weier, Electromagnetically driven convection suitable for mass transfer enhancement in liquid metal batteries, Appl. Therm. Eng. 143, 293 (2018).

[14] P. Personnettaz, S. Landgraf, M. Nimtz, N. Weber, and T. Weier, Mass transport induced asymmetry in charge/discharge behavior of liquid metal batteries, Electrochem. Commun. 105, 106496 (2019).

[15] W. Herreman, C. Nore, P. Ziebell Ramos, L. Cappanera, J.-L. Guermond, and N. Weber, Numerical simulation of electrovortex flows in cylindrical fluid layers and liquid metal batteries, Phys. Rev. Fluids 4, 113702 (2019).

[16] R. A. Khairulin, R. N. Abdullaev, S. V. Stankus, A. S. Agazhanov, and I. V. Savchenko, Volumetric properties of Lithium-Lead melts, Int. J. Thermophys. 38, 23 (2016).

[17] J. Saar and H. Ruppersberg, Calculation of $C_{p}(T)$ for liquid Li/Pb alloys from experimental $\rho(T)$ and $(\delta p / \delta T)_{s}$ data, J. Phys. F: Met. Phys. 17, 305 (1987).

[18] Y. Jiang, T. Cao, P. Song, D. Zhang, Y. Shi, and N. Cai, Effects of magnetically induced flow on electrochemical reacting processes in a liquid metal battery, J. Power Sources 438, 226926 (2019).

[19] J.-L. Guermond, R. Laguerre, J. Léorat, and C. Nore, An interior penalty Galerkin method for the MHD equations in heterogeneous domains, J. Comput. Phys. 221, 349 (2007).

[20] J.-L. Guermond, R. Laguerre, J. Léorat, and C. Nore, Nonlinear magnetohydrodynamics in axisymmetric heterogeneous domains using a Fourier/finite element technique and an interior penalty method, J. Comput. Phys. 228, 2739 (2009).

[21] A. Jackson, A. Sheyko, P. Marti, A. Tilgner, D. Cébron, S. Vantieghem, R. Simitev, F. Busse, X. Zhan, G. Schubert, S. Takehiro, Y. Sasaki, Y.-Y. Hayashi, A. Ribeiro, C. Nore, and J.-L. Guermond, A spherical shell numerical dynamo benchmark with pseudo-vacuum magnetic boundary conditions, Geophys. J. Int. 196, 712 (2013).

[22] P. Marti, N. Schaeffer, R. Hollerbach, D. Cébron, C. Nore, F. Luddens, J.-L. Guermond, J. Aubert, S. Takehiro, Y. Sasaki, Y.-Y. Hayashi, R. R. Simitev, F. Busse, S. Vantieghem, and A. Jackson, Full sphere hydrodynamic and dynamo benchmarks, Geophys. J. Int. 197, 119 (2014).

[23] L. Cappanera, J.-L. Guermond, W. Herreman, and C. Nore, Momentum-based approximation of incompressible multiphase fluid flows, Int. J. Numer. Methods Fluids 86, 541 (2018). 
[24] W. Herreman, C. Nore, L. Cappanera, and J.-L. Guermond, Tayler instability in liquid metal columns and liquid metal batteries, J. Fluid Mech. 771, 79 (2015).

[25] W. Herreman, C. Nore, J.-L. Guermond, L. Cappanera, N. Weber, and G. M. Horstmann, Perturbation theory for metal pad roll instability in cylindrical reduction cells, J. Fluid Mech. 878, 598 (2019).

[26] W. Gąsior and Z. Moser, Thermodynamic study of liquid lithium-lead alloys using the EMF method, J. Nucl. Mater. 294, 77 (2001).

[27] H. S. Carslaw and J. C. Jaeger, Conduction of Heat in Solids, 2nd ed. (Clarendon Press, Oxford, 1959).

[28] V. Bojarevičs, J. A. Freibergs, E. I. Shilova, and E. V. Shcherbinin, Electrically Induced Vortical Flows, Mechanics of Fluids and Transport Processes (Springer Netherlands, 1989).

[29] V. Bojarevics, J. A. Freibergs, E. I. Shilova, and E. V. Shcherbinin, Electrically Induced Vortical Flows (Kluwer Academic, Amsterdam, 1989).

[30] M. Wanschura, H. C. Kuhlmann, and H. J. Rath, Three-dimensional instability of axisymmetric buoyant convection in cylinders heated from below, J. Fluid Mech. 326, 399 (1996).

[31] H. Kim, D. A. Boysen, J. M. Newhouse, B. L. Spatocco, B. Chung, P. J. Burke, D. J. Bradwell, K. Jiang, A. A. Tomaszowska, K. Wang, W. Wei, L. A. Ortiz, S. A. Barriga, S. M. Poizeau, and D. R. Sadoway, Liquid metal batteries: Past, present, and future, Chem. Rev. 113, 2075 (2013).

[32] B. Schulz, Thermophysical properties of the Li(17)Pb(83)alloy, Fusion Eng. Des. 14, 199 (1991).

[33] E. M. De les Valls, L. Sedano, L. Batet, I. Ricapito, A. Aiello, O. Gastaldi, and F. Gabriel, Lead-lithium eutectic material database for nuclear fusion technology, J. Nucl. Mater. 376, 353 (2008).

[34] R. A. Khairulin, S. V. Stankus, and R. N. Abdullaev, Interdiffusion in Lithium-Lead melts, Thermophys. Aeromech. 24, 773 (2017).

[35] D. Martelli, A. Venturini, and M. Utili, Literature review of lead-lithium thermophysical properties, Fusion Eng. Des. 138, 183 (2019).

[36] R. B. Bird, W. E. Stewart, and E. N. Lightfoot, Transport Phenomena (John Wiley \& Sons, New York, 2002). 